\title{
Chebyshev and fast decreasing polynomials *
}

\author{
Vilmos Totik Tamás Varga
}

January 8, 2015

\section{Contents}

1 Introduction $\quad 2$

2 Polynomials with small norms $\quad 3$

3 The main technical tool $\quad 4$

4 Proof of Theorems 2.1 and $2.2 \quad 12$

5 Fast decreasing polynomials at corners 18

6 Proof of Theorems $\mathbf{5 . 1}$ and $\mathbf{5 . 2} 20$

Step 1. Proof of the second inequality in (6.9) when $\left|z_{0}+i \tau\right| \geq d \tau 24$

Step 2. Proof of the second inequality in (6.9) for $\left|z_{0}+i \tau\right| \leq \bar{d} \tau$. 26

Proof of Lemma 6.6 for $\beta=1 \ldots \ldots \ldots$. . . . . . . . 27

Proof of Lemma 6.6 for $\beta<1 \ldots \ldots 31$

7 Proof of Theorem 5.3, Remark 5.4 and Example 5.5 34

8 Proof of Theorem $7.1 \quad 39$

9 Nikolskii type inequalities for area measures 45

10 Markov type inequalities for area measures 48

11 Christoffel functions $\quad 52$

Abstract

Extending a classical result of Widom from 1969, polynomials with small supremum norms are constructed for a large family of compact sets $\Gamma$ : their norm is at most a constant times the theoretical lower limit

*AMS subject classification: 31A15, 41A10, 41A17

Key words: Chebyshev polynomials, compact sets, corners, fast decreasing polynomials, Green's functions, conformal maps, potential theory, polynomial inequalities 
$\operatorname{cap}(\Gamma)^{n}$, where $\operatorname{cap}(\Gamma)$ denotes logarithmic capacity. The construction is based on a discretization of the equilibrium measure, and the polynomials have the additional property that outside the given set $\Gamma$ they increase as fast as possible, namely as $\operatorname{cap}(\Gamma)^{n} \exp \left(n g_{\overline{\mathbf{C}} \backslash \Gamma}(z)\right)$, with the Green's function with pole at infinity in the exponent. This latter fact allows us to use these polynomials as building blocks in constructing Dirac-delta type polynomials around corners: if a compact set $K$ has a corner at some point $z_{0}$, then Dirac-delta type polynomials (fast decreasing polynomials) peaking at $z_{0}$ are polynomials $P_{n}(z)$ with $P_{n}\left(z_{0}\right)=1$ that decrease as $\left|P_{n}(z)\right| \prec \exp \left(-n^{\beta}\left|z-z_{0}\right|^{\gamma}\right)$ on the set $K$ as $z$ moves away from $z_{0}$. The possible $(\beta, \gamma)$ pairs are completely described in turn of the angle $\alpha \pi$ at $z_{0}(\beta<1$ and $\gamma \geq \beta /(2-\alpha)$ or $\beta=1$ and $\gamma>\beta /(2-\alpha))$. As application of these fast decreasing polynomials sharp Nikolskii and Markov type inequalities are proven for Jordan domains with corners. The paper uses distortion properties of conformal maps, potential theoretic techniques as well as the theory of weighted logarithmic potentials.

\section{Introduction}

In this paper we extensively use potential theoretic concepts such as logarithmic capacity, Green's function, equilibrium measure etc., see [4], [8], [20] or [21] for these concepts and their properties.

Let $\Gamma$ be a compact subset of the complex plane consisting of infinitely many points. The Chebyshev polynomials $T_{n}(z)=z^{n}+\cdots$ associated with $\Gamma$ are the extremal polynomials that minimize the supremum norm

$$
\left\|T_{n}\right\|_{\Gamma}=\sup _{z \in \Gamma}\left|T_{n}(z)\right| .
$$

Because of their extremality they appear in many problems from number theory to numerical analysis, see for various connections the survey article [23].

It is classical (see e.g. [20, Theorem 5.5.4]) that for any $n$ and any monic polynomial $P_{n}(z)=z^{n}+\cdots$ we have

$$
\left\|P_{n}\right\|_{\Gamma} \geq \operatorname{cap}(\Gamma)^{n}
$$

and for the minimum of the left hand side we have the Fekete-Szegö-Zygmund theorem

$$
\left\|T_{n}\right\|^{1 / n} \rightarrow \operatorname{cap}(\Gamma)
$$

where $\operatorname{cap}(\Gamma)$ denotes the logarithmic capacity of $\Gamma$.

It is a highly non-trivial problem of primary importance how close one can get with the norm $\left\|P_{n}\right\|$ in (1.1) to the theoretical lower limit cap $(\Gamma)^{n}$. In the influential paper [31] H. Widom proved asymptotics and upper bounds for the

Chebyshev polynomials, in particular, his results imply that if $\Gamma$ consists of finitely many (disjoint) smooth Jordan curves and arcs, then there are polynomials $P_{n}(z)=z^{n}+\cdots$ with

$$
\left\|P_{n}\right\|_{\Gamma} \leq C \operatorname{cap}(\Gamma)^{n}
$$


for some $C$, i.e. in this case the Chebyshev numbers $\left\|T_{n}\right\|_{\Gamma}$ are at most a constant times the theoretical lower bound $\operatorname{cap}(\Gamma)^{n}$. A similar estimate if $\Gamma$ is the union of finitely many (disjoint) quasiconformal Jordan curves and arcs has been proven in the recent work [3]. If there are at least two components or $\Gamma$ is a smooth single arc, then the better estimate

$$
\left\|P_{n}\right\|_{\Gamma}=(1+o(1)) \operatorname{cap}(\Gamma)^{n}
$$

is impossible for all $n$ (see [28, Theorem 2], [31]). It is a delicate problem (connected with simultaneous Diophantine approximation of the harmonic measures of the components of $\Gamma$ ) how close (along a subsequence of the natural numbers n) $\left\|T_{n}\right\|_{K}$ can get to $\operatorname{cap}(\Gamma)^{n}$, see [27] and [28].

This paper has several goals. On the first hand, in the next sections we prove a very general extension of Widom's theorem, namely we show that (1.2) is true for a large family of sets. Then, in Sections 5-8 we apply the results from the first part of the paper to settle the problem on the existence of fast decreasing polynomials at a corner of a set. In turn, those fast decreasing polynomials will be used in Sections 9 and 10 to find the correct order in Nikolskii and Markov type inequalities with respect to area measures.

\section{Polynomials with small norms}

For a compact set $\Gamma$ let $\Omega$ denote the unbounded component of the complement $\overline{\mathbf{C}} \backslash \Gamma$. Then $\partial \Omega$ is called the outer boundary of $\Gamma$ (in what follows $\partial H$ denotes the boundary of the set $H$ ). By the maximum principle the supremum norms of polynomials on $\Gamma$ and on the outer boundary $\partial \Omega$ are the same.

A Jordan arc $\gamma$ on the complex plane (i.e. a homeomorphic image of $[0,1]$ ) is called Dini-smooth if it has a parametrization $\gamma(t), t \in[0,1]$, such that $\gamma(t)$ is differentiable, $\gamma^{\prime}(t) \neq 0$, and the modulus of continuity

$$
\omega\left(\gamma^{\prime}, \delta\right)=\sup \left\{\left|\gamma^{\prime}(t)-\gamma^{\prime}(u)\right|:|t-u| \leq \delta, t, u \in[0,1]\right\}
$$

of $\gamma^{\prime}$ satisfies

$$
\int_{0}^{1} \frac{\omega\left(\gamma^{\prime}, t\right)}{t} d t<\infty .
$$

The definition of a Dini-smooth Jordan curve (i.e the homeomorphic image of the unit circle) is similar, see [19, Section 3.3]. If we require that $\omega\left(\gamma^{\prime}, t\right) \leq C t^{\varepsilon}$ for some $\varepsilon>0$, then we say that $\gamma$ is $C^{1+}$ smooth.

Theorem 2.1 Let $\Gamma$ be a compact set such that its outer boundary is a finite union of Dini-smooth Jordan arcs that are disjoint except perhaps for their endpoints, and assume that $\Gamma$ does not have external cusps (i.e. $\Omega$ does not have an outward cusp). Then there is a constant $C$ and for every $n=1,2, \ldots$ there are monic polynomials $P_{n}(z)=z^{n}+\cdots$ of degree $n$ such that

$$
\left\|P_{n}\right\|_{\Gamma} \leq C \operatorname{cap}(\Gamma)^{n}
$$




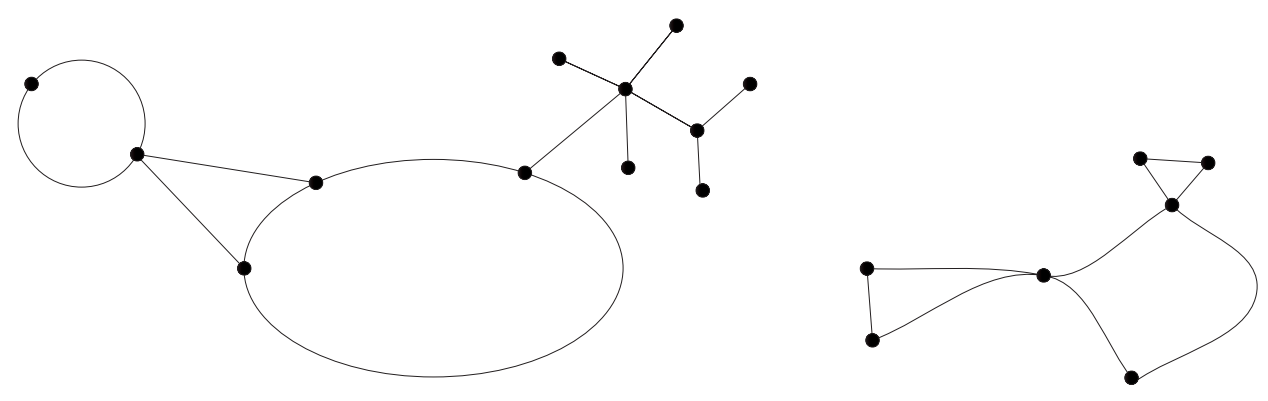

Figure 1: A typical $\Gamma$, where the dots indicate the endpoints of the arcs that build up (the outer boundary of) $\Gamma$.

Figure 1 shows a typical set for which the theorem can be applied.

Let $g_{\overline{\mathbf{C}} \backslash \Gamma}$ denote the Green's function of $\Omega$ with pole at infinity. The BernsteinWalsh lemma ([30, p. 77] or [20, Theorem 5.5.7, p. 156]) says that for polynomials $P_{n}$ of degree at most $n$ the following inequality holds:

$$
\left|P_{n}(z)\right| \leq\left\|P_{n}\right\|_{\Gamma} e^{n g_{\overline{\mathbf{C}} \backslash \Gamma}(z)}, \quad z \in \Omega .
$$

In particular, for the polynomials from (2.1) we have

$$
\left|P_{n}(z)\right| \leq C \operatorname{cap}(\Gamma)^{n} e^{n g_{\overline{\mathbf{C} \backslash \Gamma}}(z)}, \quad z \in \Omega .
$$

It is remarkable, and that will be the foundation for the results in Sections 5-8, that the $P_{n}$ 's in Theorem 2.1 can be constructed in such a way that on certain curves emanating from $\Gamma$ a matching lower bound can be given, i.e. on those curves the polynomials $P_{n}$, besides being asymptotically minimal on $\Gamma$, grow at as fast a rate as possible along those curves. To have a precise statement, let $\partial \Omega$ be the union of the Dini-smooth $\operatorname{arcs} \gamma_{j}, 1 \leq j \leq k_{0}$, which are disjoint except for their endpoints.

Theorem 2.2 With the assumptions of Theorem 2.1 the polynomials in Theorem 2.1 can be selected in such a way that besides (2.1) they also satisfy the following property. Let $E$ be an endpoint of one of the $\gamma_{j}$ 's, and let $\sigma$ be a smooth Jordan arc in $\Omega$ emanating from $E$ such that $\sigma$ is not tangent to any of the arcs $\gamma_{j}$. Then there is a constant $c=c_{\sigma}>0$ such that

$$
\left|P_{n}(z)\right| \geq c \cdot \operatorname{cap}(\Gamma)^{n} e^{n g_{\overline{\mathbf{C}} \backslash \Gamma}(z)}, \quad z \in \sigma .
$$

\section{The main technical tool}

Let $s=s_{\gamma}$ denote the arc measure on an arc (or unions of arcs) $\gamma$.

For a measure $\nu$ let

$$
U^{\nu}(z)=\int \log \frac{1}{|z-t|} d \nu(t)
$$


be its logarithmic potential. Theorem 2.1 will easily follow from the following.

In what follows, $F \sim G$ means that $\frac{1}{C} F \leq G \leq C F$ with some constant $C$.

Proposition 3.1 Let $\gamma$ be a single Dini-smooth Jordan arc with endpoints A, B, and decompose $\gamma$ into two subarcs $J_{A}$ resp. $J_{B}$ (without common interior) that contain A resp. B. Assume that $d \mu(t)=\omega(t) d s_{\gamma}(t)$ is a measure on $\gamma$ of total mass $\theta>0$ such that $\omega$ is continuous inside $\gamma$ and for some $\alpha, \beta>0$ we have

$$
\begin{array}{ll}
\omega(t) & \sim|t-A|^{\frac{1}{\alpha}-1}, \quad t \in J_{A}, \\
\omega(t) & \sim|t-B|^{\frac{1}{\beta}-1}, \quad t \in J_{B} .
\end{array}
$$

Then there is a constant $C$ and for every $n$ there are monic polynomials $P_{[n \theta]}$ of degree $[n \theta]$ such that

$$
\left|P_{n}(z)\right| \exp \left(n U^{\mu}(z)\right) \leq C, \quad z \in \gamma .
$$

Furthermore, the same statement is true for some monic polynomials $\tilde{P}_{n}$ of degree $[n \theta]+1$.

The last statement is clear if we set $\tilde{P}_{n}(z)=z P_{n}(z)$.

Proof of Proposition 3.1. Divide $\gamma$ into $[n \theta]$ arcs $I_{j}$, each having equal weight $\theta /[n \theta]$ with respect to $\mu$, i.e. $\mu\left(I_{j}\right)=\theta /[n \theta]$. Then

$$
\left|\frac{\theta}{[n \theta]}-\frac{1}{n}\right|=\left|\mu\left(I_{j}\right)-1 / n\right| \leq C / n^{2} .
$$

Let

$$
\xi_{j}=\frac{1}{\mu\left(I_{j}\right)} \int_{I_{j}} u d \mu(u)
$$

be the center of mass with respect to $\mu$, and consider the polynomial

$$
P_{n}(z)=\prod_{j}\left(z-\xi_{j}\right)
$$

of degree $[n \theta]$.

Before we embark on the proof of Proposition 3.1 we need

Proposition 3.2 If $E(=A$ or $B)$ is one of the endpoints of $\gamma$, say $E=A$, $E \in I_{1}$ and $I_{1}, I_{2}, \ldots$ follow one another in this order on $\gamma$, then $\left|\xi_{j}-E\right| \sim$ $(j / n)^{\alpha}$ and $s\left(I_{j}\right) \sim j^{\alpha-1} / n^{\alpha}$ in $J_{A}$. Furthermore, if the endpoints of the arc $I_{j}$ are $a_{j}, b_{j}$ then

$$
\left|\xi_{j}-a_{j}\right| \sim\left|\xi_{j}-b_{j}\right| \sim s\left(I_{j}\right) \sim j^{\alpha-1} / n^{\alpha},
$$

and

$$
\left|\xi_{j}-\xi_{i}\right| \sim \frac{\left|j^{\alpha}-i^{\alpha}\right|}{n^{\alpha}} .
$$

Of course, on the arc $J_{B}$ similar estimates are true with $\alpha$ replaced by $\beta$. 
Proof. Let $I_{j}$ be the arc $\widehat{a_{j} b_{j}}$ with $a_{j}$ lying closer to $E$. Then, by the assumption,

$$
j \frac{\theta}{[n \theta]}=\int_{\widehat{E b_{j}}} \omega(t) d s(t) \sim \int_{\widehat{E b_{j}}}|t-E|^{\frac{1}{\alpha}-1} d s(t),
$$

and since $|t-E| \sim s(\widehat{E t})$, we can continue this as

$$
\int_{\widehat{E b_{j}}} s(\widehat{E t})^{\frac{1}{\alpha}-1} d s(t) \sim s\left(\widehat{E b_{j}}\right)^{1 / \alpha} \sim\left|E-b_{j}\right|^{1 / \alpha} .
$$

Therefore, $\left|E-b_{j}\right| \sim(j / n)^{\alpha}$ and $s\left(I_{1}\right) \sim 1 / n^{\alpha}$ follows because $\theta /[n \theta] \sim 1 / n$. Since $a_{j}=b_{j-1}$, we also get for $j \geq 2$ the relation $\left|E-a_{j}\right| \sim(j / n)^{\alpha}$. Therefore, for $j \geq 2$

$$
\frac{\theta}{[n \theta]}=\int_{\widehat{a_{j} b_{j}}} \omega(t) d s(t) \sim \int_{\widehat{a_{j} b_{j}}}\left((j / n)^{\alpha}\right)^{\frac{1}{\alpha}-1} d s(t) \sim s\left(I_{j}\right)(j / n)^{1-\alpha}
$$

which, in view again of $\theta /[n \theta] \sim 1 / n$, gives $s\left(I_{j}\right) \sim j^{\alpha-1} / n^{\alpha}$.

Since $\xi_{j}$ lies close to $I_{j},\left|\xi_{j}-E\right| \sim(j / n)^{\alpha}$ is immediate for $j \geq 2$. To prove it for $j=1$ we may assume that $E=0$ and $\mathbf{R}_{+}$is the half-tangent to the $\operatorname{arc} \gamma$ at $E$. Let the vertical projection of the arc $I_{1}$ onto the real line be $[0, d]$. Then $d \sim 1 / n^{\alpha}$ is immediate from our previous estimates, and $\Re \xi_{1}$ is the center of mass of a measure $\rho(t) d t$ on $[0, d]$ for which $\rho(t) \sim t^{\frac{1}{\alpha}-1}(\rho$ is the vertical projection of $\mu$ onto $\mathbf{R}_{+}$). Elementary estimate shows then that $\Re \xi_{1} / d$ is bounded away from 0 and infinity (no matter how small $d$ is), which, combined with $\operatorname{diam}\left(I_{1}\right) \sim 1 / n^{\alpha}$, yields the desired estimate $\left|\xi_{1}\right| \sim(1 / n)^{\alpha}$.

The same argument verifies (3.7), while (3.8) follows from the other statements in the proposition: for example if $i<j \leq 2 i, i \neq j$ then

$$
\left|\xi_{j}-\xi_{i}\right| \sim s\left(\widehat{a_{i} b_{j}}\right)=\sum_{\tau=i}^{j} s\left(I_{\tau}\right) \sim \sum_{\tau=i}^{j}\left(\tau^{\alpha-1} / n^{\alpha}\right) \sim\left(j^{\alpha}-i^{\alpha}\right) / n^{\alpha},
$$

on the other hand if $j>2 i$ then (use also the preceding estimate with $j=2 i$ )

$$
\left|\xi_{j}-\xi_{i}\right| \sim\left|E-\xi_{j}\right| \sim j^{\alpha} / n^{\alpha} \sim\left(j^{\alpha}-i^{\alpha}\right) / n^{\alpha} .
$$

After this let us return to the proof of Proposition 3.1.

It easily follows from the assumptions on $\omega$ that

$$
\int|\log | z-t|| d \mu(t) \leq C, \quad z \in \gamma
$$


We can write

$$
\begin{aligned}
-n U^{\mu}(z) & =\sum_{j}\left(n-\frac{1}{\mu\left(I_{j}\right)}\right) \int_{I_{j}} \log |z-t| d \mu(t) \\
& +\sum_{j} \frac{1}{\mu\left(I_{j}\right)} \int_{I_{j}} \log |z-t| d \mu(t)=\Sigma_{1}+\Sigma_{2} .
\end{aligned}
$$

Here, by (3.4) and (3.9),

$$
\left|\Sigma_{1}\right| \leq \sum_{j} O(1)\left|\int_{I_{j}} \log \right| z-t|d \mu(t)|=O(1) .
$$

Therefore, to prove the claim we have to show that on $\gamma$

$$
\log \left|P_{n}(z)\right|-\Sigma_{2}=\sum_{j} \frac{1}{\mu\left(I_{j}\right)} \int_{I_{j}} \log \left|\frac{z-\xi_{j}}{z-t}\right| \omega(t) d s(t) \leq C .
$$

The proof uses the idea of [21, Theorem VI.4.2]. Thus, let $z$ lie in an $\operatorname{arc} I_{j_{0}}$ that lies, say, in $J_{A}$, and enumerate the $\operatorname{arcs} I_{j}$ in such a ways that they follow each other in the order $I_{1}, \ldots, I_{j_{0}}, \ldots$ with $I_{1}$ containing $E:=A$. $z$ and $j_{0}$ will always have this meaning below. We consider the sum

$$
\sum_{j \neq j_{0}} \frac{1}{\mu\left(I_{j}\right)} \int_{I_{j}} \log \left|\frac{z-\xi_{j}}{z-t}\right| \omega(t) d s(t)=: \sum_{j \neq j_{0}} L_{j}(z),
$$

and prove that it is uniformly bounded (both from below and above). Note that this sum differs from the one on the right of (3.12) in one term (the term with integral over $I_{j_{0}}$ is missing), and we shall actually show that not just the sum, but also the sum consisting of the absolute values $\left|L_{j}\right|$ is uniformly bounded, i.e.

$$
\sum_{j \neq j_{0}}\left|L_{j}(z)\right|=O(1)
$$

First we verify that the individual terms $L_{j}(z)$ in (3.13) are uniformly bounded on $\gamma$. The uniform boundedness of $L_{j}(z)$ is clear for $j \neq j_{0} \pm 1$ (the $j=j_{0}$ term is not in the sum), for then in the integrand

$$
\left|z-\xi_{j}\right| \sim \operatorname{dist}\left\{I_{j_{0}}, I_{j}\right\} \sim|z-t| \quad \text { for all } t \in I_{j} .
$$

So let $j=j_{0} \pm 1$, and consider first $j=j_{0}+1$. Then we know from Proposition 3.2 that $\left|z-\xi_{j_{0}+1}\right| \sim s\left(I_{j_{0}+1}\right) \sim\left(j_{0}+1\right)^{\alpha-1} / n^{\alpha}$, and from the assumption that $\omega(t) \leq C\left(n /\left(j_{0}+1\right)\right)^{\alpha-1}$ on $I_{j_{0}+1}$ (note that for $t \in I_{j_{0}+1}$ we have $|t-A| \sim$ $\left.\left(\left(j_{0}+1\right) / n\right)^{\alpha}\right)$. Let $I_{j_{0}+1}$ be the arc $\widehat{a b}$, see Figure 2. Clearly

$$
\begin{aligned}
L_{j_{0}+1}(z) & =\frac{1}{\mu\left(I_{j_{0}+1}\right)} \int_{I_{j_{0}+1}} \log \left|\frac{z-\xi_{j_{0}+1}}{z-t}\right| \omega(t) d s(t) \\
& \leq C n\left(\frac{n}{j_{0}+1}\right)^{\alpha-1} \int_{I_{j_{0}+1}}\left(\log \left|z-\xi_{j_{0}+1}\right|+\log \frac{1}{|a-t|}\right) d s(t) .
\end{aligned}
$$




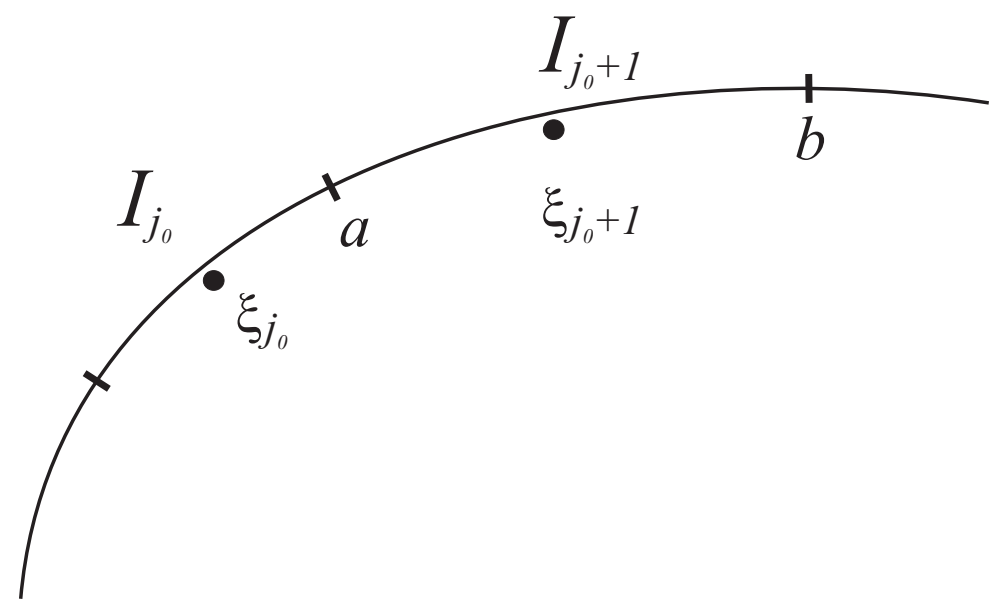

Figure 2: The position of $z, a, b$

Here

$\int_{I_{j_{0}+1}} \log \frac{1}{|a-t|} d s(t) \leq \int_{I_{j_{0}+1}} \log \frac{C_{0}}{s(\widehat{a} t)} d s(t)=s\left(I_{j_{0}+1}\right)\left(\log C_{0}+1-\log s\left(I_{j_{0}+1}\right)\right)$.

Therefore, the integral on the right of (3.15) equals

$$
s\left(I_{j_{0}+1}\right) \log \frac{\left|z-\xi_{j_{0}+1}\right|}{s\left(I_{j_{0}+1}\right)}+O\left(s\left(I_{j_{0}+1}\right)\right) \leq C s\left(I_{j_{0}+1}\right) \leq C \frac{\left(j_{0}+1\right)^{\alpha-1}}{n^{\alpha}} .
$$

If we substitute this into (3.15) then we obtain the boundedness of $L_{j_{0}+1}(z)$ from above. Its boundedness from below is clear since for $z \in I_{j_{0}}, t \in I_{j_{0}+1}$ we have

$$
\left|\frac{z-\xi_{j_{0}+1}}{z-t}\right| \geq c>0
$$

by (3.7). This proves the uniform boundedness of the individual terms $L_{j}$, $j \neq j_{0}$.

The case $j=j_{0}-1$ is completely similar when $j_{0}-1 \neq 1$. When $j=$ $j_{0}-1=1$ then $\omega(t) \leq C\left(n /\left(j_{0}-1\right)\right)^{\alpha-1}$ is no longer true. In this case (i.e. when $\left.I_{j_{0}-1}=I_{1}=: \widehat{a b}\right)$ we have $\mu\left(I_{1}\right) \sim 1 / n \sim s\left(I_{1}\right)^{1 / \alpha},|z-t| \sim s(\widehat{z t})$, so

$$
L_{j_{0}-1}=L_{1} \leq \frac{C}{s(\widehat{a b})^{1 / \alpha}} \int_{\widehat{a b}} \log \frac{C s(\widehat{a b})}{s(\widehat{z t})} s(\widehat{a t})^{\frac{1}{\alpha}-1} d s(t),
$$

and the right-hand side will be shown to be bounded from above in the proof of (3.21) (the boundedness of $L_{1}$ from below is again a consequence of (3.16)).

It follows from Proposition 3.2 that there is an $M$ such that if $\left|j-j_{0}\right| \geq M$ then for $z \in I_{j_{0}}$ and $t \in I_{j}$ we have

$$
\left|\frac{\xi_{j}-t}{z-\xi_{j}}\right| \leq \frac{1}{2}
$$


Thus, in this case for the integrands in $L_{j}(z)$ we get (use that with any local branch of the logarithm we have $\log |1-u|=\Re \log (1-u))$

$$
\begin{aligned}
\log \left|\frac{z-\xi_{j}}{z-t}\right|=-\log \left|1+\frac{\xi_{j}-t}{z-\xi_{j}}\right| & =-\Re \log \left(1+\frac{\xi_{j}-t}{z-\xi_{j}}\right) \\
& =-\Re \frac{\xi_{j}-t}{z-\xi_{j}}+O\left(\left|\frac{\xi_{j}-t}{z-\xi_{j}}\right|^{2}\right) .
\end{aligned}
$$

Therefore, for such $j$ we have

$$
\left|L_{j}(z)\right|=\frac{1}{\mu\left(I_{j}\right)} \int_{I_{j}} O\left(\left|\frac{\xi_{j}-t}{z-\xi_{j}}\right|^{2}\right) d \mu(t)=O\left(\frac{s\left(I_{j}\right)^{2}}{\left|\xi_{j}-\xi_{j_{0}}\right|^{2}}\right)
$$

because the integral

$$
\int_{I_{j}} \Re \frac{\xi_{j}-t}{z-\xi_{j}} d \mu(t)=\Re \frac{1}{z-\xi_{j}} \int_{I_{j}}\left(\xi_{j}-t\right) d \mu(t)
$$

vanishes by the choice of $\xi_{j}$.

The expression on the right of (3.17) is bounded by a constant times $s\left(I_{j}\right)^{2}$ when $I_{j}$ is far from $I_{j_{0}}$ (say farther than a fixed constant $\delta>0$ ), and for $I_{j}$ close to $I_{j_{0}}$ (closer than $\delta$ ) it is at most (see Proposition 3.2) a constant times

$$
\frac{s\left(I_{j}\right)^{2}}{\left|j^{\alpha} / n^{\alpha}-j_{0}^{\alpha} / n^{\alpha}\right|^{2}} \sim \frac{\left(j^{\alpha-1} / n^{\alpha}\right)^{2}}{\left|j^{\alpha} / n^{\alpha}-j_{0}^{\alpha} / n^{\alpha}\right|^{2}}=\frac{j^{2 \alpha-2}}{\left|j^{\alpha}-j_{0}^{\alpha}\right|^{2}} .
$$

All in all, if we take into account the uniform boundedness of the terms we obtain that the sum in (3.14) is at most

$$
\begin{aligned}
\sum_{\left|j-j_{0}\right| \leq M, j \neq j_{0}}\left|L_{j}\right| & +\sum_{\left|j-j_{0}\right|>M}\left|L_{j}\right| \\
& \leq(2 M) C+C \sum_{\left|j-j_{0}\right|>M} \frac{j^{2 \alpha-2}}{\left|j^{\alpha}-j_{0}^{\alpha}\right|^{2}}+C \sum_{j} s\left(I_{j}\right)^{2} \leq C .
\end{aligned}
$$

Indeed, the last but one sum can be broken into three parts:

- for the sum of those $j$ 's with $j<j_{0} / 2$, in which case $j^{2 \alpha-2} /\left|j^{\alpha}-j_{0}^{\alpha}\right|^{2} \sim$ $j^{2 \alpha-2} / j_{0}^{2 \alpha}$,

- for the sum of those $j$ 's with $j_{0} / 2<j<2 j_{0}$ in which case $j^{2 \alpha-2} / \mid j^{\alpha}-$ $\left.j_{0}^{\alpha}\right|^{2} \sim 1 /\left(j-j_{0}\right)^{2}$

- and for the sum with $j \geq 2 j_{0}$, in which case $j^{2 \alpha-2} /\left|j^{\alpha}-j_{0}^{\alpha}\right|^{2} \sim 1 / j^{2}$, and each of these sums are bounded by a constant. 
To complete the proof of the proposition we have to show that the additional term

$$
\frac{1}{\mu\left(I_{j_{0}}\right)} \int_{I_{j_{0}}} \log \left|\frac{z-\xi_{j_{0}}}{z-t}\right| \omega(t) d s(t)
$$

in (3.12) is also bounded from above (from below we cannot claim boundedness for $z$ can be very close to $\xi_{j_{0}}$ ). As before, we get from Proposition 3.2 that for $j_{0}>1$ this term is at most

$$
C n \int_{I_{j_{0}}}\left(\log \frac{C s\left(I_{j_{0}}\right)}{s(\widehat{z t})}\right)\left(\frac{j_{0}^{\alpha}}{n^{\alpha}}\right)^{\frac{1}{\alpha}-1} d s(t),
$$

which, with $I_{j_{0}}=: \widehat{a b}$, equals

$$
C \frac{n^{\alpha}}{j_{0}^{\alpha-1}}(s(\widehat{a b}) \log (C s(\widehat{a b}))-s(\widehat{z b}) \log s(\widehat{z b})-s(\widehat{a z}) \log s(\widehat{a z})+s(\widehat{a b})) .
$$

Now we use that for $0 \leq x \leq y \leq 1$ the inequality

$$
-(x+y) \log 2 \leq x \log x+y \log y-(x+y) \log (x+y) \leq 0
$$

is true, and apply this with $s(\widehat{z b}), s(\widehat{a z})$ in place of $x, y$ (in which case $x+y=$ $s(\widehat{a b}))$ to continue (3.19) as

$$
\leq C \frac{n^{\alpha}}{j_{0}^{\alpha-1}}(s(\widehat{a b}) \log (C s(\widehat{a b}))-s(\widehat{a b}) \log s(\widehat{a b})+O(s(\widehat{a b}))) \leq C \frac{n^{\alpha}}{j_{0}^{\alpha-1}} s(\widehat{a b}) \leq C,
$$

where, in the last step we used that, by Proposition 3.2, $s(\widehat{a b})=s\left(I_{j_{0}}\right) \sim$ $j_{0}^{\alpha-1} / n^{\alpha}$. This gives the required estimate for (3.18) for $j_{0}>1$.

When $j_{0}=1$ then $E$ is an endpoint of the $\operatorname{arc} I_{j_{0}}$, e.g. $E=a$. In that case $\omega$ is not bounded on $I_{j_{0}}$, so we have to proceed differently than before. Similarly as above, now we have with $s(\widehat{a b})=s\left(I_{j_{0}}\right) \sim 1 / n^{\alpha}$ and $\mu\left(I_{1}\right) \sim 1 / n \sim s(\widehat{a b})^{1 / \alpha}$ the bound

$$
\frac{C}{s(\widehat{a b})^{1 / \alpha}} \int_{\widehat{a b}} \log \frac{C s(\widehat{a b})}{s(\widehat{z t})} s(\widehat{a t})^{\frac{1}{\alpha}-1} d s(t)=: I
$$

for the expression in (3.18). Recall that $z$ lies on the arc $\widehat{a b}=\widehat{E b}$, and let $w$ be the midpoint on the arc $\widehat{E z}$ in the sense that $s(\widehat{E w})=s(\widehat{w z})$, see Figure 3. Now we split the integral in (3.21) over $\widehat{a b}$ into three parts: the integrals over $\widehat{z b}, \widehat{w z}$ and $\widehat{E w}$. For the first we have for the case when $\alpha \geq 1$ the estimate (use that the antiderivative of $t^{1 / \alpha-1} \log t$ is $\left.\alpha t^{1 / \alpha} \log t-\alpha^{2} t^{1 / \alpha}\right)$

$$
\begin{aligned}
& \int_{\widehat{z b}} \log \frac{C s(\widehat{a b})}{s(\widehat{z t})} s(\widehat{a t})^{\frac{1}{\alpha}-1} d s(t) \leq \int_{\widehat{z b}} \log \frac{C s(\widehat{a b})}{s(\widehat{z t})} s(\widehat{z t})^{\frac{1}{\alpha}-1} d s(t) \\
& =\alpha \log (C s(\widehat{a b})) s(\widehat{z b})^{1 / \alpha}-\alpha s(\widehat{z b})^{1 / \alpha} \log s(\widehat{z b})+\alpha^{2} s(\widehat{z b})^{1 / \alpha} \leq C s(\widehat{a b})^{1 / \alpha}
\end{aligned}
$$




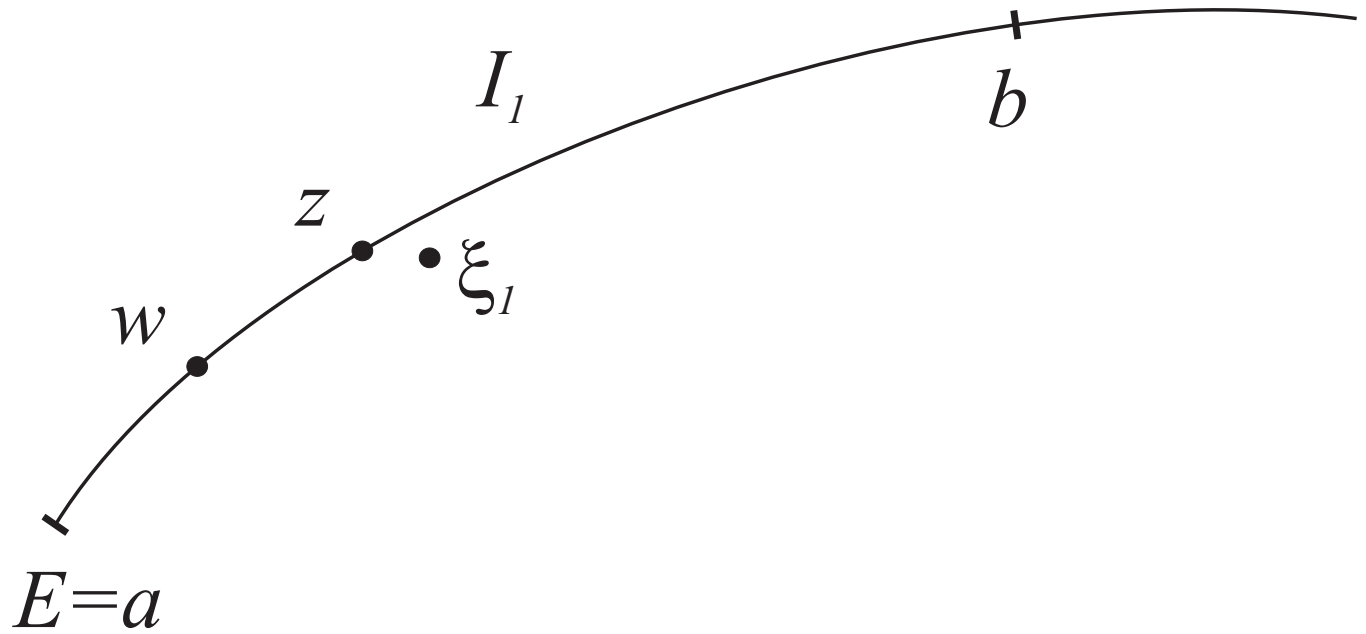

Figure 3: The choice of $w$

because for $C_{0}>e^{\alpha}$, which we may assume, we have (by the monotonicity of $x^{1 / \alpha} \log C / x$ on $\left.(0,1)\right)$

$$
\alpha s(\widehat{z b})^{1 / \alpha} \log \frac{C s(\widehat{a b})}{s(\widehat{z b})} \leq \alpha s(\widehat{a b})^{1 / \alpha} \log \frac{C s(\widehat{a b})}{s(\widehat{a b})}=\alpha s(\widehat{a b})^{1 / \alpha} \log C .
$$

This gives the estimate for the integral over $\widehat{z b}$ for $\alpha \geq 1$. When $0<\alpha<1$ then in (3.22) we cannot replace $s(\widehat{a t})^{\frac{1}{\alpha}-1}$ by $s(\widehat{z t})^{\frac{1}{\alpha}-1}$, but we can replace it by $s(\widehat{a b})^{\frac{1}{\alpha}-1}$ to get for the integral in question the bound

$$
\begin{aligned}
C s(\widehat{a b})^{\frac{1}{\alpha}-1} \int_{\widehat{z b}} \log \frac{C s(\widehat{a b})}{s(\widehat{z t})} d s(t) & \leq C s(\widehat{a b})^{\frac{1}{\alpha}-1}\left(s(\widehat{z b}) \log \frac{C s(\widehat{a b})}{s(\widehat{z b})}+s(\widehat{z b})\right) \\
& \leq C s(\widehat{a b})^{1 / \alpha}
\end{aligned}
$$

by the monotonicity of $t \log (C / t)$ on $(0,1)$.

The integral over $\widehat{w z}$ can be similarly handled. Finally, for the integral over $\widehat{E w}$ we have the bound

$$
\begin{aligned}
\int_{\widehat{E w}} \log \frac{C s(\widehat{a b})}{s(\widehat{a w})} s(\widehat{a t})^{\frac{1}{\alpha}-1} d s(t) & \leq \log \frac{C s(\widehat{a b})}{s(\widehat{a w})} \alpha s(\widehat{a w})^{1 / \alpha} \leq \log \frac{C s(\widehat{a b})}{s(\widehat{a b})} \alpha s(\widehat{a b})^{1 / \alpha} \\
& =\alpha(\log C) s(\widehat{a b})^{1 / \alpha} .
\end{aligned}
$$

Substituting all these into (3.21) we get

$$
I \leq C,
$$


and with this the upper boundedness of (3.18) for $j_{0}=1$, as well.

Before closing this section we give a lower bound for the polynomials $P_{n}$ constructed above. Fix a small $\varepsilon>0$, and with the notations used in the proof let $\Delta_{j}(\varepsilon)$ be the disk of radius $\varepsilon j^{\alpha-1} / n^{\alpha}$ about $\xi_{j}$ when $I_{j}$ lies in $J_{A}$, while in the opposite case let $\Delta_{j}(\varepsilon)$ be the disk of radius $\varepsilon j^{\beta-1} / n^{\beta}$ about $\xi_{j}$.

Proposition 3.3 The $P_{n}$ constructed in the proof of Proposition 3.1 also satisfy

$$
\left|P_{n}(z)\right| \exp \left(n U^{\mu}(z)\right) \geq c_{\varepsilon}, \quad z \in \partial\left(\gamma \cup \bigcup_{j} \Delta_{j}(\varepsilon)\right)
$$

with some constant $c_{\varepsilon}>0$.

Proof. Let first $z \in \partial \Delta_{j_{0}}(\varepsilon)$, and assume that $I_{j_{0}} \subset J_{A}$.

In view of (3.11)-(3.14) we have for $z \in \Delta_{j_{0}}(\varepsilon) \cap \gamma$

$$
\log \left|P_{n}(z)\right|+n U^{\mu}(z)=O(1)+\frac{1}{\mu\left(I_{j_{0}}\right)} \int_{I_{j_{0}}} \log \left|\frac{z-\xi_{j_{0}}}{z-t}\right| \omega(t) d s(t)
$$

where the $O(1)$ is uniform in $n$ and $j_{0}$. A closer inspection of the proof reveals that the same estimate holds on the whole $\Delta_{j_{0}}(\varepsilon)$, as well (note that the disk $\Delta_{j_{0}}(\varepsilon)$ about $\xi_{j_{0}}$ has diameter $2 \varepsilon j_{0}^{\alpha-1} / n^{\alpha}$, and for small $\varepsilon$ this is much smaller than the distance from any point of $\Delta_{j_{0}}$ to the endpoints of the arcs $I_{j_{0}}$, see Proposition 3.2). Thus, to prove (3.24) for $z \in \partial \Delta_{j_{0}}(\varepsilon)$ all we need to do is to prove the lower boundedness of the integral term in (3.25). But that is clear, since for $z \in \partial \Delta_{j_{0}}(\varepsilon)$ and $t \in I_{j_{0}}$ we have, in view of Proposition 3.2,

$$
\left|\frac{z-\xi_{j_{0}}}{z-t}\right| \geq \frac{c \varepsilon j_{0}^{\alpha-1} / n^{\alpha}}{s\left(I_{j_{0}}\right)+\varepsilon j_{0}^{\alpha-1} / n^{\alpha}} \geq c_{1}>0
$$

with some constant $c_{1}$ independent of $j_{0}$ and $n$ (which may however depend on $\varepsilon)$.

The argument is similar if $z \in \gamma \backslash \bigcup_{j} \Delta_{j}(\varepsilon)$. Indeed, if, say, $z \in I_{j_{0}}$, then (3.25) is true (see (3.11)-(3.14)), and the lower boundedness of the integral term in (3.25) follows again from (3.26).

\section{Proof of Theorems 2.1 and 2.2}

Proof of Theorem 2.1. Since the capacity of $\Gamma$ coincides with the capacity of its outer boundary, we may assume that $\Gamma$ coincides with its outer boundary (if (2.1) is true on the outer boundary, then it is true on $\Gamma$ ). As before, let $\Gamma$ be 


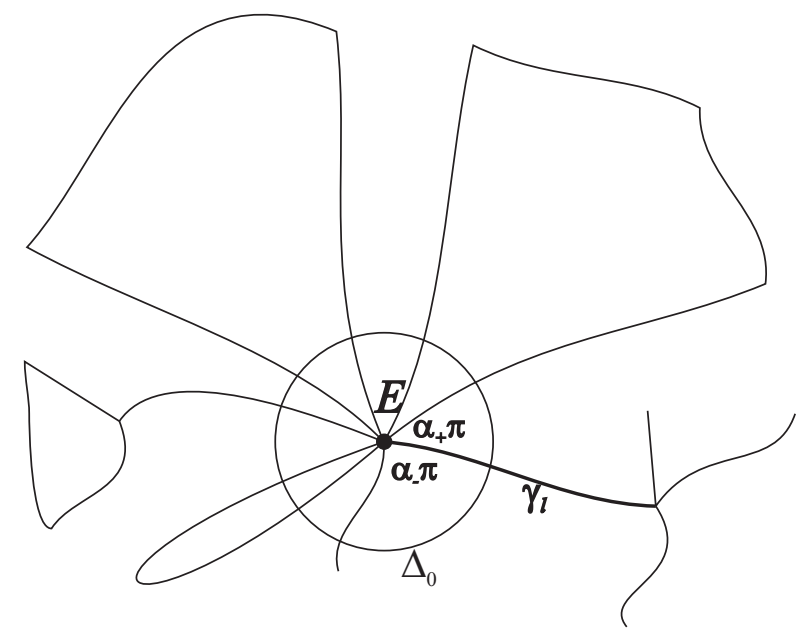

Figure 4: $\gamma_{l}$ and the angles $\alpha_{ \pm} \pi$. In this case $\alpha=\max \alpha_{ \pm}=\alpha_{-}$

the union of the Dini-smooth $\operatorname{arcs} \gamma_{j}, 1 \leq j \leq k_{0}$, which are disjoint except for their endpoints. Note that $\Gamma$ can have many (but only finitely many) multiple points (where the $\gamma_{j}$ 's meet), and at a multiple point it can have several external angles, but none of them can be 0 .

Let $\theta_{j}=\mu_{\Gamma}\left(\gamma_{j}\right)$, and for an $n$ consider integers $n_{j}=\left[\theta_{j} n\right]$ or $\left[\theta_{j} n\right]+1$, $j=1, \ldots, k_{0}$ so that $\sum_{j=1}^{k_{0}} n_{j}=n$.

Next, we need to estimate the density of the equilibrium measure. To this end let $E$ be one of the endpoints of one of the $\operatorname{arcs} \gamma_{l}$. This $E$ may belong to several other $\operatorname{arcs} \gamma_{j}$, which cut a small closed neighborhood $\Delta_{0}$ of $E$ into several sectors, see Figure 4 . Some of these sectors lie in $\Omega$, some lie in one of the connected components of $\overline{\mathbf{C}} \backslash \Gamma$; we only consider the former ones and call them external sectors. There is one or two external sectors that contain $\Delta_{0} \cap \gamma_{l}$ on its boundary. If there is only one sector with angle (seen from $\Omega$ ) equal to $\alpha \pi$ then we set $\alpha_{l}=\alpha$, and if there are two such external sectors with angles $\alpha_{-} \pi$ and $\alpha_{+} \pi$, then we set $\alpha_{l}=\max \left(\alpha_{-}, \alpha_{+}\right)$. Note that since external cusps are not allowed, this $\alpha_{l}$ is positive.

Lemma 4.1 Under the assumptions of Theorem 2.1 $d \mu_{\Gamma}=\omega_{\Gamma}(t) d s_{\Gamma}(t)$, where the density $\omega_{\Gamma}$ is continuous away from the endpoints of the arcs $\gamma_{j}, j=$ $1, \ldots, k_{0}$. If $E$ is an endpoint of one of the $\gamma_{j}$ 's, say of $\gamma_{l}$, then in a neighborhood of $E$ the ratio $\omega_{\Gamma}(t) /|t-E|^{1 / \alpha_{l}-1}$ is continuous and positive on $\gamma_{l}$.

A more precise formulation of the last statement is that $\omega_{\Gamma}(t) /|t-E|^{1 / \alpha_{l}-1}$, $t \neq E$ is continuous on $\gamma_{l}$ in a neighborhood of $E$, and it has positive and finite limit (along $\gamma_{l}$ ) at $t=E$.

Let $\mu_{j}$ be the restriction of the equilibrium measure $\mu_{\Gamma}$ to $\gamma_{j}$. The lemma shows that we can apply Proposition 3.1 to each $\gamma_{j}, \mu_{j}, \theta_{j}$ and $n$ (replacing $\gamma$, 
$\mu, \theta, n$ in the proposition), and we get monic polynomials $P_{n, j}$ of exact degree $n_{j}$ such that

$$
\left|P_{n, j}(z)\right| \exp \left(n U^{\mu_{j}}(z)\right)=\left|P_{n, j}(z)\right| \exp \left(U^{n \mu_{j}}(z)\right) \leq C_{j}, \quad z \in \gamma_{j} .
$$

Here $n \mu_{j}$ has total mass $n \theta_{j}$, while $\left(n_{j} / \theta_{j}\right) \mu_{j}$ has total mass $n_{j}$, which is either $\left[n \theta_{j}\right]$ or $\left[n \theta_{j}\right]+1$. Therefore $n \mu_{j}-\left(n_{j} / \theta_{j}\right) \mu_{j}=\rho_{j, n} \mu_{j}$ with $-1 \leq \rho_{j, n} \leq 1$. Since $U^{\mu_{\Gamma}}$ is uniformly bounded on compact subsets of the complex plane, the same is true of each $U^{\mu_{j}}$, which implies the same for

$$
\exp \left(U^{\left(n_{j} / \theta_{j}\right) \mu_{j}-n \mu_{j}}\right) .
$$

This, together with (4.1) show that

$$
\left|P_{n, j}(z)\right| \exp \left(U^{\left(n_{j} / \theta_{j}\right) \mu_{j}}(z)\right) \leq C_{j}, \quad z \in \gamma_{j} .
$$

But

$$
\log \left(\left|P_{n, j}(z)\right| \exp \left(U^{\left(n_{j} / \theta_{j}\right) \mu_{j}}(z)\right)\right)
$$

is subharmonic on $\overline{\mathbf{C}} \backslash \gamma_{j}$ including the point infinity where it is harmonic, so the maximum principle gives that (4.2) is actually true throughout the complex plane. Now we can multiply the inequalities (4.2) together for all $j=1,2, \ldots, k_{0}$ to conclude with $P_{n}=\prod_{j} P_{n, j}$ of degree precisely $n$ that

$$
\left|P_{n}(z)\right| \exp \left(\sum_{j} U^{\left(n_{j} / \theta_{j}\right) \mu_{j}}(z)\right) \leq C, \quad z \in \mathbf{C} .
$$

According to the preceding argument we can replace on the left each measure $\left(n_{j} / \theta_{j}\right) \mu_{j}$ by $n \mu_{j}$ to get

$$
\left|P_{n}(z)\right| \exp \left(\sum_{j} U^{n \mu_{j}}(z)\right) \leq C, \quad z \in \mathbf{C},
$$

i.e.

$$
\left|P_{n}(z)\right| \exp \left(n U^{\mu_{\Gamma}}(z)\right) \leq C, \quad z \in \mathbf{C} .
$$

But for $z \in \Gamma$ we have

$$
U^{\mu_{\Gamma}}(z)=\log \frac{1}{\operatorname{cap}(\Gamma)},
$$

so the claim in Theorem 2.1 follows from (4.3).

Proof of Theorem 2.2. We use the setup from the preceding proof, as well as the disks $\Delta_{k}(\varepsilon)$ from Proposition 3.3 for all of the arcs $\gamma_{j}$. According to that proposition

$$
\left|P_{n, j}(z)\right| \exp \left(U^{n \mu_{j}}(z)\right) \geq c_{j}, \quad z \in \partial\left(\gamma_{j} \cup \bigcup_{k} \Delta_{k}(\varepsilon)\right),
$$


where the union on the right is for those disks $\Delta_{k}(\varepsilon)$ that are created for the arc $\gamma_{j}$ (for small $\varepsilon>0$ these are precisely those disks $\Delta_{k}(\varepsilon)$ that intersect $\gamma_{j}$ ).

Arguing as in the preceding proof, we obtain

$$
\left|P_{n, j}(z)\right| \exp \left(U^{\left(n_{j} / \theta_{j}\right) \mu_{j}}(z)\right) \geq c_{j}, \quad z \in \partial\left(\gamma_{j} \cup \bigcup_{k} \Delta_{k}(\varepsilon)\right) .
$$

The logarithm of the left-hand side is harmonic in $\overline{\mathbf{C}} \backslash\left(\gamma_{j} \cup \bigcup_{k} \Delta_{k}(\varepsilon)\right)$ (including the point infinity), hence (4.5) is actually true throughout $\mathbf{C} \backslash\left(\gamma \cup \bigcup_{k} \Delta_{k}(\varepsilon)\right)$ by the maximal principle. Here we can again replace each measure $\left(n_{j} / \theta_{j}\right) \mu_{j}$ by $n \mu_{j}$ to conclude

$$
\left|P_{n, j}(z)\right| \exp \left(n U^{\mu_{j}}(z)\right) \geq c_{j}, \quad z \in \mathbf{C} \backslash\left(\Gamma \cup \bigcup_{k} \Delta_{k}(\varepsilon)\right),
$$

where now we take the union on the right for all disks $\Delta_{k}(\varepsilon)$ created for all the $\operatorname{arcs} \gamma_{j}$. On multiplying these inequalities together we get for the product $P_{n}=\prod P_{n, j}$ of exact degree $n$

$$
\left|P_{n}(z)\right| \exp \left(n U^{\mu_{\Gamma}}(z)\right) \geq c, \quad z \in \mathbf{C} \backslash\left(\Gamma \cup \bigcup_{k} \Delta_{k}(\varepsilon)\right) .
$$

Since (see e.g. [20, Sec. 4.4] or [21, (I.4.8)])

$$
U^{\mu_{\Gamma}}(z)=\log \frac{1}{\operatorname{cap}(\Gamma)}-g_{\overline{\mathbf{C}} \backslash \Gamma}(z),
$$

to complete the proof all we need to mention that for sufficiently small $\varepsilon>0$ the curve $\sigma$ in Theorem 2.2 lies in the set $\mathbf{C} \backslash\left(\Gamma \cup \bigcup_{k} \Delta_{k}(\varepsilon)\right)$ because it is not tangent to any of the $\gamma_{j}$ 's (c.f. also Proposition 3.2).

We still need to prove Lemma 4.1.

Proof of Lemma 4.1. First of all, note that the Green's function $g_{\overline{\mathbf{C}} \backslash \Gamma}$ is continuous on $\mathbf{C}$ by Wiener's criterion [20, Theorem 5.4.1].

First let $J$ be a closed subarc on $\Gamma$ not containing any of the endpoints of the $\operatorname{arcs} \gamma_{j}$. Let $G$ be a simply connected domain with Dini-smooth boundary that lies in the unbounded component of $\overline{\mathbf{C}} \backslash \Gamma$ such that $J$ lies on the boundary of $G$, and let $\Phi$ be a conformal map from the unit disk $\Delta$ onto $G$. If both sides of $J$ belong to $\partial \Omega$ then we choose such a $G$ for both sides, and do the following for both of them.

By the [19, Theorem 3.5]) this $\Phi$ can be extended to a continuously differentiable function to the closed unit disk and $\Phi^{\prime}$ has a nonzero derivative there. 
The function $h(z)=g_{\overline{\mathbf{C} \backslash \Gamma}}(\Phi(z))$ is harmonic in $\Delta$ and continuous on the closed unit disk, so we have Poisson's formula for it:

$$
h\left(r e^{i \theta}\right)=\frac{1}{2 \pi} \int_{-\pi}^{\pi} \frac{1-r^{2}}{1-2 r \cos (t-\theta)+r^{2}} h\left(e^{i t}\right) d t .
$$

If $J^{\prime}$ is the arc of the unit circle that is mapped by $\Phi$ into $J$, then $h\left(e^{i t}\right)=0$ on $J^{\prime}$, so it follows from (4.7) that $h$ (considered as a function on the closed unit disk) is $C^{\infty}$ on any closed subarc of the interior of $J^{\prime}$. Hence $g_{\overline{\mathbf{C}} \backslash \Gamma}(z)=h\left(\Phi^{-1}(z)\right)$ is a $C^{1}$-function on any closed subarc of the interior of $J$. Furthermore, (4.7) gives also that

$$
h\left(r e^{i t}\right) \geq \frac{1-r}{1+r} \frac{1}{2 \pi} \int_{-\pi}^{\pi} h\left(e^{i t}\right) d t=\frac{1-r}{1+r} h(0)>0,
$$

which gives via the mapping $\Phi$

$$
g_{\overline{\mathbf{C}} \backslash \Gamma}(z+t \mathbf{n}) \geq c t
$$

for any $z \in J$ with a positive constant $c>0$ depending only on $G$, where $\mathbf{n}$ is the normal to $\gamma$ at $z$ in the direction of $G$. Hence

$$
\frac{g_{\overline{\mathbf{C}} \backslash \Gamma}}{\partial \mathbf{n}}(z) \geq c, \quad z \in J .
$$

Now all we need to do is to cite the formula [17, II.(4.1)] (or apply [21, I.(4.8)] and [21, Theorem II.1.5], which are valid also in the Dini smooth case considered here) according to which then in the interior of $J$ we have

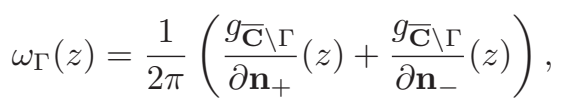

where $\mathbf{n}_{ \pm}$are the two normals to $\Gamma$ at $z$. The continuity of $\omega_{\Gamma}$ on $J$ follows from the $C^{1}$ smoothness of $g_{\overline{\mathbf{C}} \backslash \Gamma}$ there, while the positivity is a consequence of (4.8) (where $\mathbf{n}$ is one of $\mathbf{n}_{ \pm}$and note also that both normal derivatives in (4.9) are nonnegative - of course, the normal derivative with respect to a normal pointing into a bounded component of $\overline{\mathbf{C}} \backslash \Gamma$ is 0 ).

Next, let $E \in \gamma_{l}$ be an endpoint of the arc $\gamma_{l}$, and consider one of the external sectors $S$ attached to $\gamma_{l}$ of angle $\alpha \pi, 0<\alpha \leq 2$, and let this angle be enclosed by the arcs $\gamma_{l}$ and $\gamma_{l_{1}}\left(l_{1}=l\right.$ is possible). Let again $G$ be a domain lying in the sector $S \subset \Omega$ such that $G$ has on its boundary the part of $\gamma_{l} \cup \gamma_{l_{1}}$ that lies in the disk $\{|z-E| \leq \rho\}$, with some $\rho>0$, and except for the corner at $E$ of opening $\alpha \pi$, the boundary of $G$ is Dini-smooth, see Figure 5 . Let again $\Phi$ be a conformal map from the unit disk onto $G$ such that 1 is mapped into $E$. For $t \in \Gamma,|t-E|<\rho$, let $\mathbf{n}$ denote the inner normal to $\partial G$ at $t$. Set $\bar{t}=\Phi^{-1}(t)$ and let $\overline{\mathbf{n}}$ be the inner normal to the unit circle at the point $\bar{t}$. By [19, Theorem 3.9] the functions (with any local branch of the powers)

$$
\frac{\Phi(z)-E}{(z-1)^{\alpha}} \text { and } \frac{\Phi^{\prime}(z)}{(z-1)^{\alpha-1}}
$$




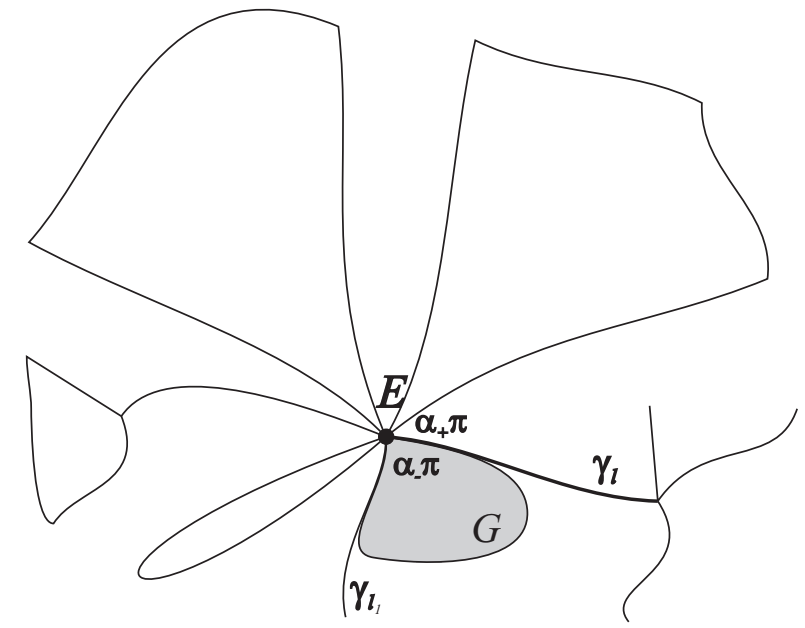

Figure 5: The domain $G\left(\alpha=\alpha_{-}\right)$

are continuous in the closed unit disk (use also [19, Theorem 3.5] when $z$ does not lie close to 1 ). Now for small $u \rightarrow 0$ the point $\bar{t}+u \overline{\mathbf{n}}$ is mapped into the point

$$
\Phi(\bar{t}+u \overline{\mathbf{n}})=t+u\left|\Phi^{\prime}(\bar{t})\right| \overline{\mathbf{n}} \frac{\Phi^{\prime}(\bar{t})}{\left|\Phi^{\prime}(\bar{t})\right|}+o(u)=t+u\left|\Phi^{\prime}(\bar{t})\right| \mathbf{n}+o(u)
$$

(use that $\Phi$ is conformal at the boundary, so $\overline{\mathbf{n}} \Phi^{\prime}(\bar{t}) /\left|\Phi^{\prime}(\bar{t})\right|$ is the normal to $\partial G$ at $t)$. Hence for $h(z)=g_{\overline{\mathbf{C}} \backslash \Gamma}(\Phi(z))$ the formula

$$
\frac{h(\bar{t}+u \overline{\mathbf{n}})-h(\bar{t})}{u}=\left|\Phi^{\prime}(\bar{t})\right| \frac{g_{\overline{\mathbf{C}} \backslash \Gamma}\left(t+u\left|\Phi^{\prime}(\bar{t})\right| \mathbf{n}+o(u)\right)-g_{\overline{\mathbf{C}} \backslash \Gamma}(t)}{u\left|\Phi^{\prime}(\bar{t})\right|}
$$

shows that

$$
\frac{\partial h(\bar{t})}{\partial \overline{\mathbf{n}}}=\left|\Phi^{\prime}(\bar{t})\right| \frac{\partial g_{\overline{\mathbf{C} \backslash \Gamma}}(t)}{\partial \mathbf{n}} .
$$

In view of the positivity and boundedness of the expressions in (4.10), here

$$
\left|\Phi^{\prime}(\bar{t})\right|=(1+o(1)) c_{1}|\bar{t}-1|^{\alpha-1} \quad \text { and } \quad \frac{|t-E|}{|\bar{t}-1|^{\alpha}}=(1+o(1)) c_{2}, \quad \text { as } t \rightarrow 1,
$$

with some constants $c_{1}, c_{2}>0$, furthermore, by the argument given in the first part of the proof, the left-hand side in (4.11) is a positive $C^{\infty}$ function around 1. All these yield

$$
\frac{\partial g_{\overline{\mathbf{C}} \backslash \Gamma}(t)}{\partial \mathbf{n}}=\frac{\partial h(\bar{E})}{\partial \overline{\mathbf{n}}}(1+o(1)) \frac{c_{2}^{1-\frac{1}{\alpha}}}{c_{1}}|t-E|^{\frac{1}{\alpha}-1}, \quad t \in \gamma_{l}, t \rightarrow E .
$$


If there is only one external sector attached to $\gamma_{l}$ at $E$, then on the other side of $\gamma_{l}$ the Green's function $g_{\overline{\mathbf{C}} \backslash \Gamma}$ is identically 0 (that side lies in one of the connected components of $\overline{\mathbf{C}} \backslash \Gamma$ ), and in that case Lemma 4.1 follows from (4.12) and (4.9).

If there are two external sectors with angles $\alpha_{ \pm} \pi$, do the aforementioned analysis for both of them to get

$$
\frac{\partial g_{\overline{\mathbf{C} \backslash \Gamma}}(t)}{\partial \mathbf{n}_{ \pm}}=\frac{\partial h(\bar{E})}{\partial \overline{\mathbf{n}}}(1+o(1)) \frac{c_{2, \pm}^{1-\frac{1}{\alpha}}}{c_{1, \pm}}|t-E|^{\frac{1}{\alpha_{ \pm}}-1}, \quad t \in \gamma_{l}, t \rightarrow E
$$

where $\mathbf{n}_{ \pm}$denote the two normals to $\gamma_{l}$ at $t$. If $\alpha \pi$ is the larger of these two external angles $\alpha_{ \pm} \pi$, then (4.13) gives, via (4.9), that $\omega_{\Gamma}(t) /|t-E|^{\frac{1}{\alpha}-1}$ has a positive limit at $E$ on $\gamma_{l}$.

\section{Fast decreasing polynomials at corners}

In this section we are going to apply Theorems 2.1 and 2.2 for constructing fast decreasing polynomials which take their absolute maximum at (or close to) a corner of a domain and exponentially decrease on the domain. We are going to give the best possible rate depending on the angle at the corner.

Fast decreasing polynomials appear in many different situations (see e.g. [10], [11], [12], [14, Theorem 7.5]) for they are particularly useful in localization and in constructing well localized "partitions of unity". As a model case consider the interval $[-1,1]$, where we are interested in polynomials $P_{n}$ of degree at most $n$ (or $\leq C n$ with some fixed $C$ ) that have the property that $P_{n}(0)=1$, and, as $x \in[-1,1]$ moves away from the origin, the polynomials decrease fast in absolute value. Two kinds of decrease have been particularly useful in applications:

(a) $\left|P_{n}(x)\right| \prec e^{-n|x|^{\beta}}, \quad x \in[-1,1]$,

(b) $\left|P_{n}(x)\right| \prec e^{-|n x|^{\gamma}}, \quad x \in[-1,1]$,

(where $A \prec B$ means that $A \leq C B$ with some constant $C$ ). From the results in the paper [10] (see [25, Theorem 4.1] and [26, Lemma 4]) it follows that (a) is possible if and only if $\beta>1$, and (b) is possible if and only if $\gamma<1$. In particular, the decrease $\left|P_{n}(x)\right| \prec e^{-n|x|}$ is not possible (for this order of decreases one would need polynomials of degree $\sim n \log n)$. In (a) the decrease is exponentially fast in $n$ at every point $x \in[-1,1], x \neq 1$, but the $n$-th polynomial starts to get small only for $x \geq 1 / n^{1 / \beta}$. In (b) the order of decrease is smaller at every $x$, but the $n$-th polynomial starts to get small for $x \geq 1 / n$, and here $1 / n$ is much smaller than $1 / n^{1 / \beta}$. We shall find the complete analogue of these results around corner points. Absolutely new techniques are needed in these cases, for no transformation will reduced the corner case to previously known results. 
Let $K$ be a compact set on the plane. In this part of the paper we may always assume that $\overline{\mathbf{C}} \backslash K$ is simply connected. Let $K$ have a Dini-smooth corner at $z_{0}$ of inner angle $\alpha \pi$. This means precisely that if $B_{\delta}\left(z_{0}\right)$ is the disk of radius $\delta$ about $z_{0}$, then for small $\delta>0$ the set $K \cap \overline{B_{\delta}\left(z_{0}\right)}$ is the closure of a Jordan domain with piecewise Dini-smooth boundary consisting of two Dinismooth Jordan arcs $J_{1}, J_{2} \subset K$ connecting $z_{0}$ with the boundary of $B_{\delta}\left(z_{0}\right)$, and of a circular arc on the boundary of $B_{\delta}\left(z_{0}\right)$. The angle of $K$ at $z_{0}$ is what $J_{1}$ and $J_{2}$ form at $z_{0}$ (with respect to $K \cap \overline{B_{\delta}\left(z_{0}\right)}$ ).

We are interested in polynomials $P_{n}$ which take the value 1 at $z_{0}$, and, as $z \in K$ moves away from $z_{0}$, the value $P_{n}(z)$ decreases as fast as possible. This decrease will be of the form $\leq D \exp \left(-d n^{\beta}\left|z-z_{0}\right|^{\gamma}\right.$ ) (with some fixed constants $D, d>0)$ and our aim is to determine what $\beta, \gamma$ are possible in terms of the angle $\alpha \pi$. Clearly, the smaller the $\gamma$ is, the fastest is the decrease.

Values $\beta>1$ are not possible at all: if for $\beta>1$ we had polynomials of this kind and $B$ is a closed disk lying in the interior of $K$, then we would have on $B$

$$
\left|P_{n}(z)\right| \leq D \exp \left(-c n^{\beta}\right)
$$

with some $c>0$. But then the Bernstein-Walsh lemma (see (6.2) below) would imply with some $C>0$

$$
\left|P_{n}\left(z_{0}\right)\right| \leq D \exp \left(-c n^{\beta}+C n\right) \rightarrow 0,
$$

which contradicts $P_{n}\left(z_{0}\right)=1$.

First we consider the $\alpha<1$ case. Then for $\beta \leq 1$ we have

Theorem 5.1 Let $K$ be a compact set on the plane with a Dini-smooth corner at $z_{0}$ of inner angle $\alpha \pi, 0<\alpha<1$. If $0<\beta \leq 1$, then for $\gamma>\frac{\beta}{2-\alpha}$ there exist constants $D, d>0$ such that for every $n$ there is a polynomial $P_{n}$ of degree at most $n$ with the following properties:

(i) $P_{n}\left(z_{0}\right)=1$,

(ii) $\left|P_{n}(z)\right| \leq D e^{-d n^{\beta}\left|z-z_{0}\right|^{\gamma}}$, and

(iii) $\left|P_{n}(z)\right| \leq 1$ on $K$.

Theorem 5.2 Under the conditions of Theorem 5.1 if $\gamma<\frac{\beta}{2-\alpha}$, then no matter what $D, d>0$ are, for large $n$ there are no polynomials of degree at most $n$ with the properties (i) and (ii).

Next, we discuss the $\gamma=\frac{\beta}{2-\alpha}$ "boundary" case. For that we need a slightly stronger assumption than Dini-smoothness, namely we need to assume that the corner at $K$ is $C^{1+\varepsilon}$ smooth for some $\varepsilon>0$, which we express by saying that the corner is $C^{1+}$ smooth.

Theorem 5.3 Assume, in addition to the assumptions of Theorem 5.1, that the corner at $z_{0}$ is $C^{1+}$ smooth. 
(a) If $\beta<1$, then for $\gamma=\frac{\beta}{2-\alpha}$ there are polynomials $P_{n}$ with properties (i) - (iii).

(b) If $\beta=1$ and $\gamma=\frac{1}{2-\alpha}$, then for any $D, d>0$ there is an $n_{0}$ such that for $n \geq n_{0}$ there are no polynomials $P_{n}$ of degree $n$ with the properties (i) and (ii).

So far we have assumed that the angle at $z_{0}$ was $\alpha \pi<\pi$. For completeness we mention the $\alpha=1$ case, which will be used in the applications in Sections 9 and 10. Note that then $\beta /(2-\alpha)$ becomes $\beta$.

Remark 5.4 Suppose that at $z_{0}$ the set $K$ has a $C^{1+}$ smooth inner angle $\pi$, and assume also that there is a disk in the complement of $K$ that contains the point $z_{0}$ on its boundary. Then all conclusions of Theorems 5.1-5.3 hold.

Theorem 5.2 is also true when $1<\alpha<2$, but that is not so for Theorem 5.1; in this case properties (i)-(iii) are not possible. Indeed, if $P_{n}\left(z_{0}\right)=1$, then the level line $\left\{z|| P_{n}(z) \mid=1\right\}$ has a subarc containing $z_{0}$ and lying in the interior of $K$, therefore $\left|P_{n}(z)\right| \leq 1$ is not possible for all $z \in K$. It is an open problem if Theorem 5.1 holds for $1<\alpha<2$ when we drop condition (iii).

In Theorem 5.3 we used $C^{1+}$ smoothness at the corner. We are going to construct an example showing that mere $C^{1}$ smoothness is not enough.

Example 5.5 Let $\alpha<1, \beta<1$. There is a $K$ which has a $C^{1}$ smooth angle at 0 of size $\alpha \pi$ such that for $\gamma=\frac{\beta}{2-\alpha}$ and for any $D, d>0$ there is an $n_{0}$ such that for $n \geq n_{0}$ there are no polynomials $P_{n}$ of degree $n$ with the properties (i) and (ii).

Theorems 5.1 and 5.2 will be proven in the next section using the results from the first part of the paper and properties of some conformal maps and Green's functions. Theorem 5.3 (along with Example 5.5) will be proven in the following two sections using the theory of weighted logarithmic potentials.

\section{Proof of Theorems 5.1 and 5.2}

We shall prove Theorem 5.1 in the equivalent form

Theorem 6.1 Let $K$ be a compact set on the plane with a Dini-smooth corner at $z_{0}$ of inner angle $\alpha \pi, 0<\alpha<1$. If $0<\beta \leq 1$, then for $\gamma>\frac{\beta}{2-\alpha}$ there exist constants $D, d>0$ such that for every $n$ there is a polynomial $P_{n}$ of degree at most dn with the following properties:

(i) $P_{n}\left(z_{0}\right)=1$,

(ii) $\left|P_{n}(z)\right| \leq D e^{-n^{\beta}\left|z-z_{0}\right|^{\gamma}}$, and

(iii) $\left|P_{n}(z)\right| \leq 1$ on $K$. 
First we deal with the case when $K$ is an isosceles triangle, and then extend the result to arbitrary $K$. We need some preliminaries concerning conform mappings.

If $K$ is compact and $\overline{\mathbf{C}} \backslash K$ is simply connected, then, by the Riemannmapping theorem, there is a unique conformal mapping $\Phi$ from $\overline{\mathbf{C}} \backslash K$ onto the exterior of the unit disk with the normalization $\Phi(\infty)=\infty$ and $\Phi^{\prime}(\infty)>0$ (cf. [6, Theorem 4.2]). If $g_{\overline{\mathbf{C} \backslash K}}(z)$ denotes Green's function of $\overline{\mathbf{C}} \backslash K$ with pole at infinity, then

$$
\log |\Phi(z)|=g_{\overline{\mathbf{C}} \backslash K}(z)
$$

(see e.g. the proof of [20, Theorem 4.4.11]). If $K$ is bounded by a Jordan curve, then $\Phi$ has a continuous and injective extension to $\overline{\mathbf{C}} \backslash K$, which we continue to denote by $\Phi$ (see Charathéodory's Theorem in [19, Theorem 2.6]).

The Green's function $g_{\overline{\mathbf{C}} \backslash K}$ will often be used in the Bernstein-Walsh lemma (6.2) ([30, p. 77] or $\left[20\right.$, Theorem 5.5 .7$, p. 156]): if $Q_{n}$ is a polynomial of degree at most $n$, then

$$
\left|Q_{n}(z)\right| \leq e^{n g_{\overline{\mathbf{C}} \backslash K}(z)}\left\|Q_{n}\right\|_{K}, \quad z \in \mathbf{C} .
$$

The following lemma states that the conformal mapping $\Phi$ possesses a kind of quasi-distance-preserving property.

Lemma 6.2 ([2, Theorem 4.1, pp. 97-98]) Suppose that $K$ has piecewise Dini-smooth boundary. Let $z_{1}, z_{2}, z_{3} \in(\mathbf{C} \backslash K) \cup \partial K$. Then the conditions $\left|\Phi\left(z_{1}\right)-\Phi\left(z_{2}\right)\right| \leq e_{1}\left|\Phi\left(z_{1}\right)-\Phi\left(z_{3}\right)\right|$ and $\left|z_{1}-z_{2}\right| \leq e_{2}\left|z_{1}-z_{3}\right|$ are equivalent; the constants $e_{1}$ and $e_{2}$ are mutually dependent but independent of $z_{1}, z_{2}, z_{3}$.

Remark 6.3 The lemma does not determine how large $e_{j}(j=1,2)$ are, so these constants can be chosen as large as we want but, of course, under a fixed bound.

For a point $z \in \partial K$ denote $\Phi^{-1}((1+\lambda) \Phi(z))$ by $\tilde{z}_{\lambda}$. A simple application of the previous lemma shows that for any $r_{1}$ there are positive constants $r_{2}=r_{2}\left(r_{1}\right)$ and $r_{3}=r_{3}\left(r_{1}\right)$ such that if $z, \zeta \in \partial K$ and $|\zeta-z| \leq r_{1}\left|\zeta-\tilde{\zeta}_{\lambda}\right|$ then

$$
r_{2} \leq \frac{\left|z-\tilde{z}_{\lambda}\right|}{\left|\zeta-\tilde{\zeta}_{\lambda}\right|} \leq r_{3}
$$

in other words $\left|\zeta-\tilde{\zeta}_{\lambda}\right| \sim\left|z-\tilde{z}_{\lambda}\right|$ (c.f. $[1,(3.5)]$ ).

Let $\Delta$ and $\Delta^{\prime}$ be similar isosceles triangles such that they are symmetric with respect to the imaginary axis, they lie in the lower half-plane, and their base is under their vertex (see Figure 6 ). Denote the vertex angle by $\alpha \pi$ with $0<\alpha<1$. Assume that the vertex of $\Delta$ is at the origin while the vertex of $\Delta^{\prime}$ is at $-\tau i(\tau>0)$. We suppose that the altitude $\Delta^{\prime}$ is 2 times as long as that of $\Delta$.

In what follows $B(z, r)=\{w:|z-w|<r\}$ denotes the open disk about $z \in \mathbf{C}$ of radius $r$. 
Consider the conformal map $\Phi$ from the complement of $\Delta^{\prime}$ onto the exterior of the unit disk. Denote its Green's level line passing through the origin by $L$, that is $L=\Phi^{-1}(\partial B(0,1+\lambda))$ for an appropriate $\lambda>0$ (see Figure 6).

Lemma 6.4 If $\tau$ is less then or equal to a suitable constant $T>0$ and $z \in$ $\Delta \backslash\left(\Delta^{\prime} \cup B(-\tau i, \tau)\right)$, then there is a constant $s>0$ independent of $\tau \in[0, T]$ such that

$$
s g_{\overline{\mathbf{C} \backslash \Delta^{\prime}}}(0) \leq\left(g_{\overline{\mathbf{C} \backslash \Delta^{\prime}}}(0)-g_{\overline{\mathbf{C} \backslash \Delta^{\prime}}}(z)\right) .
$$

Note that (6.4) claims the inequality

$$
g_{\overline{\mathbf{C} \backslash \Delta^{\prime}}}(z) \leq(1-s) \log (1+\lambda) .
$$

To prove the lemma we need an estimate for the distance between a point on the boundary of $\Delta^{\prime}$ and the level line $L$. Let $K$ be compact set with piecewise Dini-smooth boundary such that $\overline{\mathbf{C}} \backslash K$ is simply connected. Let $\zeta_{1}, \ldots, \zeta_{N}$ be the corners of $\partial K$ with angles different from $\pi$, and let $\alpha_{1} \pi, \ldots, \alpha_{N} \pi$ be the corresponding inner angles. Introduce the following function on $\partial K$ :

$$
\Theta_{\lambda}(z):= \begin{cases}\lambda^{2-\alpha_{i}} & \text { if }\left|z-\zeta_{i}\right| \leq\left|\Phi^{-1}\left((1+\lambda) \Phi\left(\zeta_{i}\right)\right)-\zeta_{i}\right| \\ \lambda \prod_{i=0}^{N}\left|z-\zeta_{i}\right|^{1-\frac{1}{2-\alpha_{i}}} & \text { otherwise. }\end{cases}
$$

With this function the following lemma is valid, in which we set $L_{\lambda}=$ $\Phi^{-1}(\partial B(0,1+\lambda))$, the $1+\lambda$-level curve of $\Phi$.

Lemma 6.5 ([29, Lemma 3.8]) If $\partial K$ is a piecewise Dini-smooth curve then there exists a constant $d_{0}=d_{0}(\partial K)$ such that

$$
\frac{1}{d_{0}} \Theta_{\lambda}(z) \leq \operatorname{dist}\left(L_{\lambda}, z\right) \leq d_{0} \Theta_{\lambda}(z)
$$

for all $z \in \partial K$.

In particular, the distance from $\zeta_{j}$ to $L_{\lambda}$ is $\sim \lambda^{2-\alpha_{j}}$, and hence the smallest distance $\rho_{\lambda}$ between $K$ and $L_{\lambda}$ satisfies

$$
\frac{1}{d_{1}} \lambda^{2-\alpha^{*}} \leq \rho_{\lambda} \leq d_{1} \lambda^{2-\alpha^{*}},
$$

where $\alpha^{*}=\min \left\{1, \alpha_{1}, \ldots, \alpha_{N}\right\}$. In view of (6.1) this implies that for any $z \notin K$ we have

$$
g_{\overline{\mathbf{C} \backslash K}}(z) \leq C \operatorname{dist}(z, K)^{\frac{1}{2-\alpha^{*}}} .
$$

For later use we record also that the same reasoning gives

$$
g_{\overline{\mathbf{C} \backslash H}}(w) \leq C \operatorname{dist}(w, H)^{\frac{1}{2-\alpha^{\prime}}}, \quad w<0,
$$

if $H$ is a triangle with vertices at $0,\left(a, \pm a \tan \left(\alpha^{\prime} \pi / 2\right)\right), a>0$, (so that at the origin $H$ has an angle equal to $\left.\alpha^{\prime} \pi\right)$. 


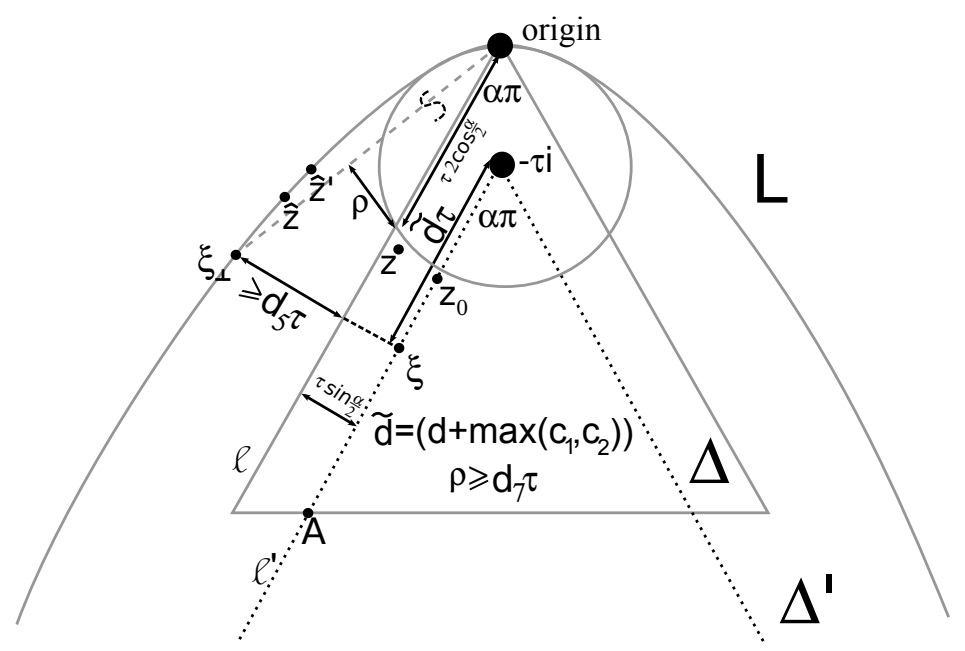

Figure 6: For explanation see the proof of Lemma 6.4.

Proof of Lemma 6.4. We introduce some notations (see Figure 6):

- $\hat{z}:=\Phi^{-1}\left((1+\lambda) \frac{\Phi(z)}{|\Phi(z)|}\right)$ - the point on the level line $L_{\lambda}=\Phi^{-1}(\partial B(0,1+$ $\lambda)$ ) corresponding to a $z \in \mathbf{C} \backslash \operatorname{Int}\left(\Delta^{\prime}\right)$.

- $z_{0}:=\Phi^{-1}\left(\frac{\Phi(z)}{|\Phi(z)|}\right)$ - the point on the boundary $\partial \Delta^{\prime}$ corresponding to a $z \in \mathbf{C} \backslash \operatorname{Int}\left(\Delta^{\prime}\right)$. Note that $\hat{z}=\left(\tilde{z_{0}}\right)_{\lambda}$.

- $\ell\left(\ell^{\prime}\right)$ denotes the leg of $\Delta\left(\Delta^{\prime}\right)$ lying on the left side of the imaginary axis.

- $z_{0}^{\perp}$ denotes the nearest point on $\ell^{\prime}$ to a point $z \in \Delta \backslash\left(\Delta^{\prime} \cup B(-\tau i, \tau)\right)$ located between $\ell$ and $\ell^{\prime}$.

- $A$ denotes the intersection of $\ell^{\prime}$ and the base of $\Delta$.

First we mention that (for sufficiently small $\tau$ ) $\Delta$ lies inside the level line $L$. Since $L$ is convex (see [18, Theorem 2.9]), this follows if we show that the leg $\ell$ lies inside the level line $L$. Indeed, let $\eta$ be the midpoint of $\ell^{\prime}$, and $\eta_{\perp}$ the intersection of the line of $\ell$ with the line that passes through $\eta$ and is perpendicular to $\ell^{\prime}$ (which is the same as being perpendicular to $\ell$ ). The computation in (6.15) below shows for $z_{0}=\eta$ that the distance from $\eta$ to $L$ is $\geq d_{5} \tau^{\frac{1}{2-\alpha}}$, and in view of $\alpha<1$, this is larger than $\tau$ for sufficiently small $\tau$. Thus, $\eta_{\perp}$ lies inside $L$, and since $L$ is convex, the same is true of the segment connecting 0 and $\eta_{\perp}$, and this last segment contains $\ell$.

We are going to prove the existence of a constant $c$ such that

$$
\frac{1}{c}|\hat{z}-z| \leq\left|\hat{z}-z_{0}\right| \leq c|\hat{z}-z|
$$


for every $z \in \Delta \backslash\left(\Delta^{\prime} \cup B(-\tau i, \tau)\right)$. Then, by Lemma 6.2 , there is a constant $1 / 2>s>0$ such that

$$
2 s \lambda=2 s\left|\Phi(\hat{z})-\Phi\left(z_{0}\right)\right| \leq|\Phi(\hat{z})-\Phi(z)|
$$

whenever $z \in \Delta \backslash\left(\Delta^{\prime} \cup B(-\tau i, \tau)\right)$.

Let $\hat{T}>0$ be so small that for every $x \in[0, \hat{T}]$

$$
(1-s) x \leq \log (1+x) \leq x
$$

hold, and choose $T$ so that, if $\tau=T$ then $|\Phi(0)|=1+\hat{T}$.

Recall that $|\Phi(\hat{z})|=1+\lambda=|\Phi(0)|$ and note that $\Phi(\hat{z}) / \Phi(z)$ is a positive real number, hence

$$
|\Phi(z)|=|\Phi(\hat{z})|-|\Phi(\hat{z})-\Phi(z)|=1+\lambda-|\Phi(\hat{z})-\Phi(z)| .
$$

Therefore, if $z \in \Delta \backslash\left(\Delta^{\prime} \cup B(-\tau i, \tau)\right)$ then, by (6.10), we have that

$$
|\Phi(z)| \leq 1+\lambda-2 s \lambda=1+(1-2 s) \lambda .
$$

So, if $\tau \leq T$ also holds, then (6.1) implies that

$$
\begin{aligned}
g_{\overline{\mathbf{C} \backslash \Delta^{\prime}}}(0)-g_{\overline{\mathbf{C} \backslash \Delta^{\prime}}}(z) & =\log |\Phi(0)|-\log |\Phi(z)| \geq(1-s) \lambda-(1-2 s) \lambda \\
& =s \lambda \geq s \log (1+\lambda)=s g_{\overline{\mathbf{C} \backslash \Delta^{\prime}}}(0)
\end{aligned}
$$

what proves (6.4).

Thus, it is left to prove (6.9). The left-hand side is easy: we only have to note that, by Lemma $6.2,\left|z-z_{0}\right| \leq d_{1}\left|\hat{z}-z_{0}\right|$ with some suitable constant $d_{1}$ (because $\left.\left|\Phi(z)-\Phi\left(z_{0}\right)\right| \leq \lambda=\left|\Phi(\hat{z})-\Phi\left(z_{0}\right)\right|\right)$. Therefore

$$
|\hat{z}-z| \leq\left|\hat{z}-z_{0}\right|+\left|z-z_{0}\right| \leq\left(1+d_{1}\right)\left|\hat{z}-z_{0}\right| .
$$

We are going to prove the second inequality in (6.9) in two steps depending if $z_{0}$ is far from ( $\geq d \tau$ with some appropriately chosen $d$ ) or close to $(\leq d \tau)$ the vertex $-i \tau$ of $\Delta^{\prime}$.

Step 1. Proof of the second inequality in (6.9) when $\left|z_{0}+i \tau\right| \geq d \tau$

Note that, by Lemma 6.2 , there is a constant $c_{1} \geq 1$ such that

$$
\left|z-z_{0}\right| \leq c_{1}\left|z-z_{0}^{\perp}\right|
$$

We verify the existence of a constant $d$ independent of $\tau$ such that if $z_{0} \in \ell^{\prime} \cap \Delta$, $\left|z_{0}-(-\tau i)\right| \geq d \tau$, then

$$
c_{1} \tau \sin \left(\frac{\alpha}{2}\right) \leq \frac{\operatorname{dist}\left(L, z_{0}\right)}{2} .
$$


Once this is done, we can write

$$
\begin{aligned}
\left|z-z_{0}\right| \leq c_{1}\left|z-z_{0}^{\perp}\right| & \leq c_{1} \tau \sin \left(\frac{\alpha}{2}\right) \leq \frac{\operatorname{dist}\left(L, z_{0}\right)}{2} \\
& \leq \frac{\left|\hat{z}-z_{0}\right|}{2} \leq \frac{|\hat{z}-z|+\left|z-z_{0}\right|}{2}
\end{aligned}
$$

that is $\left|z-z_{0}\right| \leq|\hat{z}-z|$, and therefore

$$
\left|\hat{z}-z_{0}\right| \leq|\hat{z}-z|+\left|z-z_{0}\right| \leq 2|\hat{z}-z| .
$$

To prove (6.12) we look for an appropriate $d \geq 1$. Let $\zeta_{0}:=-\tau i$ and let $\zeta_{1}, \zeta_{2}$ be the other two vertices of $\Delta^{\prime}$. The corresponding angles are denoted by $\alpha_{0} \pi, \alpha_{1} \pi, \alpha_{2} \pi$, that is $\alpha_{0}=\alpha$ and $\alpha_{1}=\alpha_{2}=(1-\alpha) / 2$ respectively. Recall that the altitude of $\Delta^{\prime}$ is 2 times as long as that of $\Delta$, therefore if the legs of $\Delta$ have a length $h$ then for any point $z_{0}$ on $\ell^{\prime}$ between the intersection point $A$ and the vertex $\zeta_{0}$ we have that

$$
\min \left(\left|z_{0}-\zeta_{1}\right|,\left|z_{0}-\zeta_{2}\right|\right) \geq h .
$$

Since $\Phi^{-1}\left((1+\lambda) \Phi\left(\zeta_{0}\right)\right)=0$, by Lemma 6.2, we have that

$$
\frac{1}{d_{2}} \tau \leq \operatorname{dist}\left(L, \zeta_{0}\right) \leq d_{2} \tau
$$

with some appropriate constant $d_{2}$. Therefore, by Lemma 6.5 , there exists a constant $d_{3}$ such that

$$
\frac{1}{d_{3}} \tau^{\frac{1}{2-\alpha}} \leq \lambda \leq d_{3} \tau^{\frac{1}{2-\alpha}} .
$$

Let now $z_{0} \in \ell^{\prime} \cap \Delta$ be such that $\left|z_{0}-\zeta_{0}\right| \geq d \tau$. From the just obtained inequality (6.14), from (6.5) and from Lemma 6.5 we get that

$$
\begin{aligned}
\operatorname{dist}\left(L, z_{0}\right) & \geq \frac{1}{d_{0} d_{3}} \tau^{\frac{1}{2-\alpha}} \prod_{i=0}^{2}\left|z_{0}-\zeta_{i}\right|^{1-\frac{1}{2-\alpha_{i}}} \\
& \geq \frac{1}{d_{0} d_{3}} \tau^{\frac{1}{2-\alpha}}(d \tau)^{1-\frac{1}{2-\alpha}} h^{1-\frac{1}{2-\alpha_{1}}} h^{1-\frac{1}{2-\alpha_{2}}}=d_{4} d^{1-\frac{1}{2-\alpha}} \tau,(6.15)
\end{aligned}
$$

where $d_{4}=h^{2-\frac{1}{2-\alpha_{1}}-\frac{1}{2-\alpha_{2}}} / d_{0} d_{3}$. Hence, the inequality in (6.12) is satisfied if

$$
d_{4} d^{1-\frac{1}{2-\alpha}} \tau \geq 2 c_{1} \tau \sin \left(\frac{\alpha}{2}\right)
$$

This proves $(6.12)$ with $d:=\left(\frac{2 c_{1}}{d_{4}} \sin \left(\frac{\alpha}{2}\right)\right)^{\frac{2-\alpha}{1-\alpha}}$. 
Step 2. Proof of the second inequality in (6.9) for $\left|z_{0}+i \tau\right| \leq d \tau$

Let now $z \in \Delta \backslash\left(\Delta^{\prime} \cup B(-\tau i, \tau)\right)$ be a point between $\ell$ and $\ell^{\prime}$ for which $\left|z_{0}-\zeta_{0}\right| \leq$ $d \tau$. Apply (6.3) with $\zeta=\zeta_{0}$ and $r_{1}=d$ to get a constant $c_{2}$ such that

$$
\left|\hat{z}-z_{0}\right| \leq c_{2}\left|\zeta_{0}-0\right|=c_{2} \tau .
$$

Let $\xi \in \ell^{\prime}$ be the point for which

$$
\left|\xi-\zeta_{0}\right|=\left(d+\max \left(c_{1}, c_{2}\right)\right) \tau=: \tilde{d} \tau
$$

and denote by $\xi_{\perp}$ the (nearer) intersection of the level line $L$ and the line which passes through $\xi$ and is perpendicular to $\ell^{\prime}$ (see Figure 6 ). Since $d+\max \left(c_{1}, c_{2}\right)>$ $d$, by Step 1, we have that

$$
\left|\xi_{\perp}-\xi\right| \geq \operatorname{dist}(L, \xi) \geq 2 c_{1} \sin \left(\frac{\alpha}{2}\right) \tau,
$$

from which it follows that

$$
\operatorname{dist}\left(\ell, \xi_{\perp}\right) \geq 2 c_{1} \sin \left(\frac{\alpha}{2}\right) \tau-\sin \left(\frac{\alpha}{2}\right) \tau=: d_{5} \tau .
$$

On the other hand, by (6.11),

$$
\left|z-z_{0}\right| \leq c_{1}\left|z-z_{0}^{\perp}\right| \leq c_{1} \sin \left(\frac{\alpha}{2}\right) \tau
$$

and $\left|z_{0}-\zeta_{0}\right| \leq d \tau$. Hence elementary geometric consideration shows that $z$ lies in the interior of the quadrilateral $\left(\zeta_{0}, \xi, \xi_{\perp}, 0\right)$. Likewise, since $\left|\hat{z}-z_{0}\right| \leq c_{2} \tau$, $\hat{z}$ is a point on the subarc of $L$ which joins $\xi_{\perp}$ to the origin and lies in the left-hand side of the imaginary axis. Because of the convexity of the level line $L$ (see [18, Theorem 2.9]) the segment connecting $\xi_{\perp}$ with the origin lies between $L$ and the leg $\ell$. Denote this segment (the dashed line in Figure 6 ) by $S$. Now, by elementary geometrical consideration (see Figure 6), we can see that

$$
|S|=\operatorname{dist}\left(\xi_{\perp}, 0\right) \geq \tau \sqrt{d_{5}^{2}+\left(\tilde{d}+\cos \left(\frac{\alpha}{2}\right)\right)^{2}}=: d_{6} \tau,
$$

and, therefore,

$$
|z-\hat{z}| \geq \operatorname{dist}(S, z) \geq \frac{2 \tau \cos \left(\frac{\alpha}{2}\right)}{d_{6} \tau} d_{5} \tau:=d_{7} \tau .
$$

Hence we obtain that

$$
\begin{aligned}
\left|\hat{z}-z_{0}\right| & \leq|\hat{z}-z|+\left|z-z_{0}\right| \leq|\hat{z}-z|+c_{1}\left|z-z_{0}^{\perp}\right| \leq|\hat{z}-z|+c_{1} \sin \left(\frac{\alpha}{2}\right) \tau \\
& \leq\left(1+\frac{c_{1} \sin (\alpha / 2)}{d_{7}}\right)|\hat{z}-z| .
\end{aligned}
$$

This verifies Step 2. 
Now we are ready to prove the existence part of Theorems 6.1 and 5.3 for isosceles triangles. This fact is formulated in the following lemma.

Lemma 6.6 Let $\Delta$ be an isosceles triangle with vertex at the origin and of vertex angle $\alpha \pi, \alpha<1$. Assume that $\gamma>\frac{1}{2-\alpha}$ if $\beta=1$ and $\gamma=\frac{\beta}{2-\alpha}$ if $0<\beta<1$. Then there exist constants $D>0$ and $d>0$ such that for every $n$ there is a polynomial $P_{n}$ of degree at most $d n$ with the following properties:

(i) $P_{n}(0)=1$,

(ii) $\left|P_{n}(z)\right| \leq D e^{-n^{\beta}|z|^{\gamma}}$, and

(iii) $\left|P_{n}(z)\right| \leq 1$.

on the set $\Delta$.

Proof. We may assume that $\Delta$ is lying in the lower half-plane and it is symmetrically situated with respect to the imaginary axis.

First we deal with the case $\beta=1$.

Proof of Lemma 6.6 for $\beta=1$

Fix $n$. Let $T$ be the same number as in Lemma 6.4 and $m=m(n):=$ $\left[\log _{2}\left(n T^{\gamma}\right)\right]$, the (lower) integer part of $\log _{2}\left(n T^{\gamma}\right)$.

Take $m$ congruent isosceles triangles $\Delta_{k}^{\prime}=\Delta_{n, k}^{\prime}$ on the lower half-plane lying symmetrically on the imaginary axis with the following properties (see Figure 7):

- they are similar to $\Delta$ and their altitude is 2 times as long as that of $\Delta$,

- the $k$-th apex is located at $-\tau_{k} i$ where

$$
\tau_{k}:=\left(\frac{2^{k}}{n}\right)^{1 / \gamma}
$$

Let $\Phi_{k}$ denote the conformal map from the exterior of $\Delta_{k}^{\prime}$ onto the exterior of the unit disk. Let $\lambda_{k}$ be the number for which $\Phi_{k}^{-1}\left(\left(1+\lambda_{k}\right) \Phi_{k}\left(-\tau_{k} i\right)\right)=0$. Applying Lemma 6.5 and Lemma 6.2 to $-\tau_{k} i$ (cf. (6.14) with $\tau=\tau_{k}, \lambda=\lambda_{k}$ ), we get that

$$
\lambda_{k}^{2-\alpha} \sim \tau_{k},
$$

where $\sim$ indicates that the ratio of the two sides lies in between two positive constants. Note that " $\sim$ " is independent of $n$ and $k$. Hence, considering that 


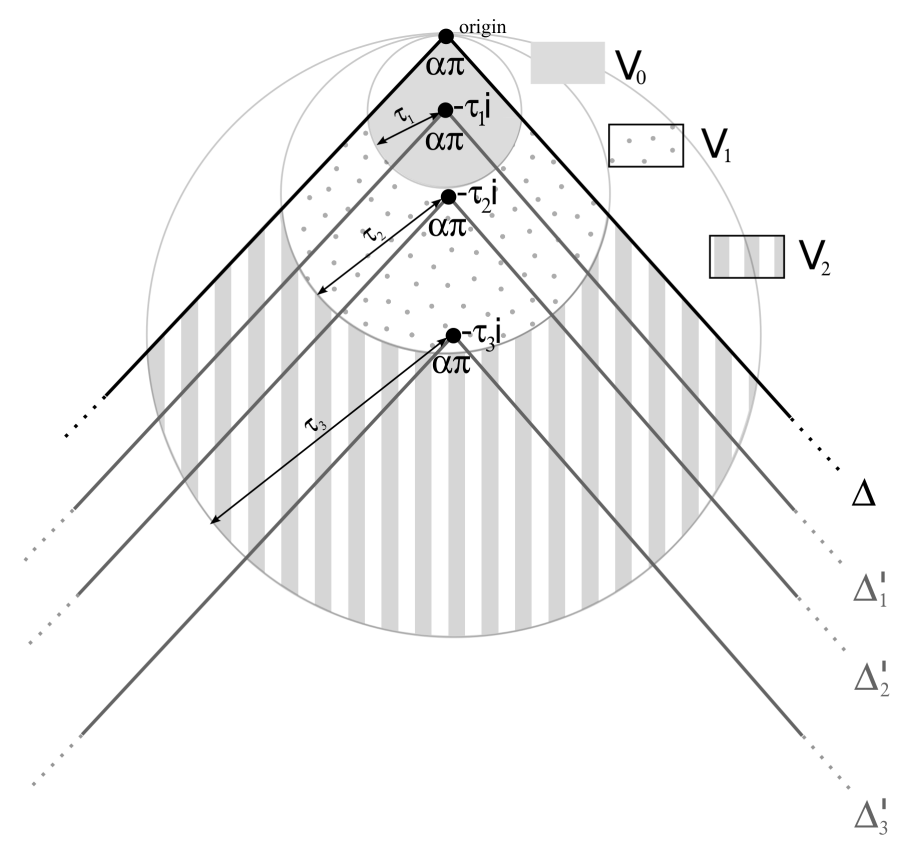

Figure 7: For explanation see the proof of Lemma 6.6.

$\left|\Phi_{k}(0)\right|=1+\lambda_{k}$ and (6.1) holds we infer the existence of a constant $c_{1}>0$ (independent of $n$ and $k$ ) such that

$$
\frac{1}{c_{1}} \tau_{k}^{\frac{1}{2-\alpha}} \leq g_{k}(0) \leq c_{1} \tau_{k}^{\frac{1}{2-\alpha}},
$$

where $g_{k}:=g_{\overline{\mathbf{C}} \backslash \Delta_{k}^{\prime}}$. Let

$$
\nu_{k}:=r \frac{2^{k}}{\tau_{k}^{1 /(2-\alpha)}},
$$

where $r$ is an appropriate constant chosen later independently of $n$ and $k$. Applying Theorems 2.1 and 2.2 to $\Delta_{k}^{\prime}$ we get a polynomial $Q_{k}$ of degree $\leq \nu_{k}$ such that

$$
\left\|Q_{k}\right\|_{\Delta_{k}^{\prime}} \leq C \operatorname{cap}\left(\Delta_{k}^{\prime}\right)^{\nu_{k}}
$$

and

$$
\left|Q_{k}(z)\right| \geq c \cdot \operatorname{cap}\left(\Delta_{k}^{\prime}\right)^{\nu_{k}} e^{\nu_{k} g_{k}(z)},
$$

whenever $z \in\left\{t i: t \in\left[0,-\tau_{k}\right]\right\}$. Note that here the constants $C, c>0$ are independent of $n$ and $k$ since the triangles $\Delta_{n, k}(n \in \mathbf{N}, 1 \leq k \leq m(n))$ are congruent. 
It is clear from the construction (see the proofs of Theorems 2.1 and 2.2) that the zeros of $Q_{k}$ lie symmetrically on the sides of $\Delta_{k}^{\prime}$ with respect to the imaginary axis.

We claim that the polynomial

$$
P_{n}(z):=\prod_{k=1}^{m} \frac{Q_{k}(z)}{Q_{k}(0)}
$$

satisfies the lemma. Clearly, $P_{n}(0)=1$.

To see (iii), investigate the polynomials $Q_{k}(z) / Q_{k}(0)$ separately. Since the zeros of $Q_{k}(z)$ lie symmetrically on the sides of $\Delta_{k}^{\prime}$, a simple geometric reasoning shows that $\left|Q_{k}(z) / Q_{k}(0)\right| \leq 1$ on $B_{k}$ where $B_{k}$ denotes the disk with center at $-\tau_{k} i$ and of radius $\tau_{k}$. (Take two zeros which are mirror images of one another onto the imaginary axis, and use that the product of the distances of these zeros from the origin is greater than or equal to the product of the distances of these zeros from a point in $B_{k}$.)

If $z \in \Delta_{k}^{\prime} \cap \Delta$ then by (6.18), (6.19) and (6.16) we get

$$
\left|\frac{Q_{k}(z)}{Q_{k}(0)}\right| \leq \frac{C \operatorname{cap}\left(\Delta_{k}^{\prime}\right)^{\nu_{k}}}{c \cdot \operatorname{cap}\left(\Delta_{k}^{\prime}\right)^{\nu_{k}} e^{\nu_{k} g_{k}(0)}} \leq \frac{C}{c} e^{-\nu_{k} \frac{1}{c_{1}} \tau_{k}^{1 /(2-\alpha)}}=\frac{C}{c} e^{-r 2^{k} / c_{1}} .
$$

This shows that if $r \geq c_{1} \log (C / c)$, then $\left|Q_{k}(z)\right| /\left|Q_{k}(0)\right| \leq 1$.

Finally, if $z \in \Delta \backslash\left(B_{k} \cup \Delta_{k}^{\prime}\right)$ then by the Bernstein-Walsh lemma (6.2) and by (6.18)-(6.19) we obtain that

$$
\left|\frac{Q_{k}(z)}{Q_{k}(0)}\right| \leq \frac{C \operatorname{cap}\left(\Delta_{k}^{\prime}\right)^{\nu_{k}} e^{\nu_{k} g_{k}(z)}}{c \cdot \operatorname{cap}\left(\Delta_{k}^{\prime}\right)^{\nu_{k}} e^{\nu_{k} g_{k}(0)}}=\frac{C}{c} e^{\nu_{k}\left(g_{k}(z)-g_{k}(0)\right)} .
$$

In view of (6.4) and (6.16) this line can be continued as

$$
\leq \frac{C}{c} e^{-\nu_{k} s g_{k}(0)} \leq \frac{C}{c} e^{-\nu_{k} s \frac{1}{c_{1}} \tau_{k}^{1 /(2-\alpha)}}=\frac{C}{c} e^{-s r 2^{k} / c_{1}}
$$

and this is less then or equal to 1 , if $r$ at least $\frac{c_{1}}{s} \log (C / c)$. Multiplying all these inequalities together we get that $\left|P_{n}(z)\right| \leq 1$ holds.

To validate (ii) we split the triangle $\Delta$ (see Figure 7):

- $V_{0}:=B_{1} \cap \Delta$

- $V_{k}:=\left(B_{k+1} \backslash B_{k}\right) \cap \Delta, 1 \leq k \leq m-1$,

- $V_{m}:=\Delta \backslash\left(V_{0} \cup \cdots \cup V_{m-1}\right)=\Delta \backslash B_{m}$.

If $z \in V_{0}$ then $|z| \leq 2 \tau_{1}$ so, using the verified property (iii), we get that

$$
\left|P_{n}(z)\right| \leq 1 \leq D e^{-n\left(2 \tau_{1}\right)^{\gamma}} \leq D e^{-n|z|^{\gamma}},
$$

if $D \geq e^{2^{1+\gamma}}$. 
If $z \in V_{k}, 1 \leq k \leq m-1$, then $2 \cos \left(\frac{\alpha \pi}{2}\right) \tau_{k} \leq|z| \leq 2 \tau_{k+1}$. Similarly to (6.21) and (6.23), we obtain that

$$
\left|P_{n}(z)\right| \leq\left|\frac{Q_{k}(z)}{Q_{k}(0)}\right| \leq \frac{C}{c} e^{-\nu_{k} s \frac{1}{c_{1}} \tau_{k}^{1 /(2-\alpha)}} .
$$

(We again used that $\left|Q_{l}(z) / Q_{l}(0)\right| \leq 1,1 \leq l \leq m$, on $\Delta$.) It is enough to see that the expression in the exponent on the right-hand side is less then or equal to $-n|z|^{\gamma}$. Note that

$$
\tau_{k+1}=\left(\frac{2^{k+1}}{n}\right)^{1 / \gamma}=2^{1 / \gamma} \tau_{k} .
$$

For the exponent we have

$$
\nu_{k} \frac{s}{c_{1}} \tau_{k}^{1 /(2-\alpha)}=r \frac{2^{k}}{\tau_{k}^{1 /(2-\alpha)}} \frac{s}{c_{1}} \tau_{k}^{1 /(2-\alpha)}=\frac{r s}{c_{1}} 2^{k} .
$$

As regards $n|z|^{\gamma}$, we estimate it as

$$
n|z|^{\gamma} \leq n\left(2 \tau_{k+1}\right)^{\gamma} \leq n\left|2^{1+1 / \gamma} \tau_{k}\right|^{\gamma}=n 2^{1+\gamma}\left(\frac{2^{k}}{n}\right)=2^{k+1+\gamma} .
$$

These show that if $r \geq \frac{c_{1}}{s} 2^{1+\gamma}$ then

$$
\nu_{k} \frac{s}{c_{1}} \tau_{k}^{1 /(2-\alpha)} \geq n|z|^{\gamma}
$$

on $V_{k}$. Therefore we can end (6.25) as

$$
\left|P_{n}(z)\right| \leq \frac{C}{c} e^{-n|z|^{\gamma}} .
$$

Finally, if $z \in V_{m}$, then

$$
\left(2 \cos \left(\frac{\alpha \pi}{2}\right)\right) T / 2^{1 / \gamma} \leq\left(2 \cos \left(\frac{\alpha \pi}{2}\right)\right) \tau_{m} \leq|z| \leq h,
$$

where $h$ is the length of the legs of $\Delta$, so we have to use the inequality $h \leq$ $2^{1 / \gamma} \frac{h}{T} \tau_{m}$ instead of $\tau_{k+1} \leq 2^{1 / \gamma} \tau_{k}$, otherwise we can do much the same as for $1 \leq k-1 \leq m$, since for such a point $z,(6.21)$ and (6.23) are also valid. Hence, we can conclude that

$$
\max \left(\frac{c_{1}}{s} \log \frac{C}{c}, 2 \frac{c_{1}}{s}, 2^{1+\gamma} \frac{c_{1}}{s},\left(8 \frac{h}{T}\right)^{\gamma} \frac{c_{1}}{s}\right)
$$

is an appropriate choice for $r$.

We still have to examine the degree of $P_{n}$. The construction of $P_{n}$ shows that

$$
\operatorname{deg}\left(P_{n}\right)=\sum_{k=1}^{m} \operatorname{deg}\left(Q_{k}\right)=\sum_{k=1}^{m} \nu_{k}=2 r \sum_{k=1}^{m} \frac{2^{k-1}}{\tau_{k}^{\frac{1}{2-\alpha}}}
$$


Noting that the rightmost side is a Riemannian sum for the function $1 /(x / n)^{1 / \gamma(2-\alpha)}$, in view of the assumption $\gamma(2-\alpha)>1$ we have

$$
\begin{aligned}
\operatorname{deg}\left(P_{n}\right) & \leq \nu_{1}+2 r \int_{2}^{2^{m}} \frac{1}{\left(\frac{x}{n}\right)^{\frac{1}{\gamma(2-\alpha)}}} \mathrm{d} x \\
& \leq n^{\frac{1}{\gamma(2-\alpha)}}\left(2^{1-1 / \gamma(2-\alpha)} r+2 r \int_{2}^{n T^{\gamma}} \frac{1}{x^{\frac{1}{\gamma(2-\alpha)}}} \mathrm{d} x\right) \leq d n .
\end{aligned}
$$

Proof of Lemma 6.6 for $\beta<1$

Recall that in this case $\gamma=\frac{\beta}{2-\alpha}$.

The proof runs as before, we only sketch the necessary changes. In this case

$$
\begin{aligned}
m=m(n) & :=\left[\beta \log _{2}\left(n T^{\frac{1}{2-\alpha}}\right)\right] \\
\tau_{k} & :=\frac{\left(2^{k}\right)^{1 / \gamma}}{n^{2-\alpha}},
\end{aligned}
$$

and

$$
\nu_{k}:=r \frac{2^{k}}{\tau_{k}^{1 /(2-\alpha)}} .
$$

Choose the triangles $\Delta_{k}^{\prime}$ and the polynomials $Q_{k}$ similarly as before and the polynomial $P_{n}$ as in (6.20). $P_{n}$ satisfies (i)-(iii), the verification of which is much the same as before.

Instead of (6.24) we should write

$$
\left|P_{n}(z)\right| \leq 1 \leq D e^{-n^{\beta}\left(2 \tau_{1}\right)^{\gamma}} \leq D e^{-n^{\beta}|z|^{\gamma}},
$$

since $z \in \Delta_{0}$, so $|z| \leq 2 \tau_{1}$. Note that the $\nu_{k}$ in terms of $\tau_{k}$ is the same as before (but $\tau_{k}$ has been changed). In particular, the reasonings leading to (6.21) and (6.23) are valid in this case, as well. Now the analogue of (6.28), namely

$$
\left|P_{n}(z)\right| \leq \frac{C}{c} e^{-n^{\beta}|z|^{\gamma}},
$$

also holds if we consider that (6.23) is true with (6.26) in the exponent, and instead of (6.27) we now have

$$
n^{\beta}|z|^{\gamma} \leq n^{\beta}\left(2^{(1 / \gamma)+1} \tau_{k}\right)^{\gamma}=2^{k+1+\gamma} .
$$

In view of $\gamma(2-\alpha)=\beta<1$ the degree of $P_{n}$ can be estimated as

$$
\operatorname{deg}\left(P_{n}\right) \leq \nu_{1}+2 r \int_{2}^{2^{m}} \frac{1}{\left(\frac{x^{1 / \gamma}}{n^{2-\alpha}}\right)^{\frac{1}{2-\alpha}}} \mathrm{d} x \leq r 2^{1-1 / \beta} n+2 r n \int_{2}^{n^{\beta} T^{\gamma}} \frac{1}{x^{\frac{1}{\beta}}} \mathrm{d} x \leq d n .
$$


Remark 6.7 Let $0<\beta<1, \varepsilon>0$ and $M_{0}, M_{1} \in \mathbf{N}$ be fixed. We shall need later that for large $n$ the inequality $\left|Q_{k}(z) / Q_{k}(0)\right| \leq 1$, that was used to prove property (iii), i.e. $\left|P_{n}(z)\right| \leq 1$ for all $z \in \Delta$, actually holds for $k \leq K_{0}$ if $z$ lies close to $\Delta$ and 0 , namely this is the case if $|z| \leq M_{0} / n^{1 /(2-\alpha)}$ and $\operatorname{dist}(z, \Delta) \leq M_{1}|z|^{1+\varepsilon}$. Indeed, if $z$ lies in the ball $B_{k}$ then this follows from the preceding proof, so assume $z \notin B_{k}$ (which can only happen for $k \leq k_{0}$ with some fixed $k_{0}$ ). Then note first of all that the distance from $z$ to the level line $L_{k}$ of $\Delta_{k}^{\prime}$ that passes through the origin is $\geq d_{0} \tau_{k}$ (with some $d_{0}$ that depends on the parameters in this remark). In fact, this is a consequence of (6.15), of $\tau_{k} \geq 1 / n^{1 /(2-\alpha)}$, and of the assumption

$$
\operatorname{dist}(z, \Delta) \leq M_{1}|z|^{1+\varepsilon} \leq C / n^{(1+\varepsilon) /(2-\alpha)} \ll d_{0} \tau_{k} .
$$

Hence, Lemma 6.4 is true for this $z$ (with a possibly different $s$ ), and then (6.22)(6.23) holds just as before, and those two formulae proved $\left|Q_{k}(z) / Q_{k}(0)\right| \leq 1$ (by choosing the $r$ in (6.23) sufficiently large).

To prove Theorem 6.1 for arbitrary $K$ we need a polynomial which is 1 at $z_{0}$ and, otherwise, less then 1 on the set $K$ in absolute value. The existence of such a polynomial comes from (e.g.) the following sharp form of Hilbert's lemniscate theorem. Recall that $\sigma \subset \mathbf{C}$ is a lemniscate if there is a polynomial $p$ such that $\sigma=\{z \in \mathbf{C}:|p(z)|=1\}$, in other words, a lemniscate is a polynomial level line.

Lemma 6.8 ([15, Theorem 1.1]) Let $\Gamma$ and $\Gamma^{\prime}$ be Jordan curves such that both of them are twice continuously differentiable in a neighborhood of a point $P$, they touch each other at $P$ and $\Gamma^{\prime} \backslash\{P\}$ lies in the interior of $\Gamma$. Assume that their curvatures at $P$ are different. Then there is a lemniscate $\sigma$ that separates $\Gamma$ and $\Gamma^{\prime}$ in the sense that

- $P \in \sigma$,

- $\sigma \backslash\{P\} \subset \operatorname{Int} L \backslash\left(\operatorname{Int} \Gamma^{\prime} \cup \Gamma^{\prime}\right)$,

where Int $\Gamma$ denotes the interior of $\Gamma$.

Proof of Theorem 6.1. If $\gamma>\frac{\beta}{2-\alpha}$, choose $1>\hat{\alpha}>\alpha$ such that

$$
\frac{\beta}{2-\hat{\alpha}}<\gamma
$$

Take an isosceles triangle $\Delta$ with vertex at $z_{0}$ and of vertex angle $\hat{\alpha} \pi$ such that the "sides" of the corner of $K$ with $\alpha \pi$ angle lies inside $\Delta$ near to $z_{0}$ (see Figure 8). Since $\hat{\alpha}>\alpha$, this is possible.

Apply Lemma 6.6 with $\hat{\alpha}$ and $\gamma$ to the triangle $\Delta$ to get a polynomial $\hat{P}_{n}$ of degree at most $\hat{d} n$ with the properties (i)-(iii) on $\Delta$. Thus, for $z \in \Delta$

$$
\left|\hat{P}_{n}(z)\right| \leq \hat{D} e^{-n^{\beta}\left|z-z_{0}\right|^{\gamma}} .
$$




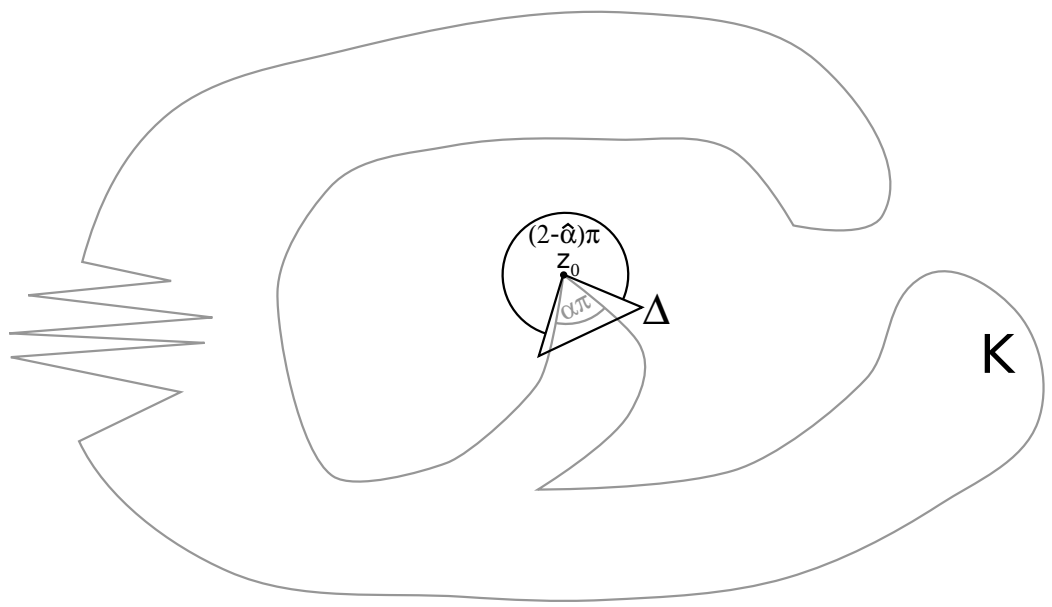

Figure 8: For explanation see the proof of Theorem 5.1.

(It can be assumed that $\left|z-z_{0}\right| \leq 1$ if $z \in \Delta$.) Denote by $M$ the supremum of $g_{\overline{\mathbf{C} \backslash \Delta}}$ on $K \backslash \Delta$. Then the Bernstein-Walsh lemma (6.2) shows that

$$
\left|\hat{P}_{n}(z)\right| \leq e^{n M}
$$

on $K$. (Remember that, by properties (i)-(iii), $\left\|\hat{P}_{n}\right\|_{\Delta}=1$.)

By Lemma 6.8 there exists a polynomial $R$ with the following properties:

- $R\left(z_{0}\right)=1$

- $\|R\|_{K}=1$, and $\|R\|_{K \backslash \Delta}=1-\varepsilon<1$.

(The reader should only consider the existence of two twice continuously differentiable Jordan curves $L, L^{\prime}$ through $z_{0}$ such that their curvatures are different at $z_{0}, L^{\prime} \backslash\left\{z_{0}\right\} \subset \operatorname{Int} L \backslash\left\{z_{0}\right\}$ and $K \backslash\left\{z_{0}\right\} \subset \operatorname{Int} L^{\prime}$.) Therefore there is an integer $m_{1}$ such that

$$
\left\|R^{m_{1}}\right\|_{K \backslash \Delta} \leq e^{-M} .
$$

On the other hand, there exists an integer $m_{2}$ such that

$$
\left\|R^{m_{2}}\right\|_{K \backslash \Delta} \leq e^{-\operatorname{diam}(K)^{\gamma}} .
$$

Now a simple calculation shows that the polynomial $P_{n}:=R^{n\left(m_{1}+m_{2}\right)} \hat{P}_{n}$ has degree at most $\left(\operatorname{deg}(R)\left(m_{1}+m_{2}\right)+\hat{d}\right) n$ and possesses the properties (i)-(iii) with respect to $K$.

Proof of Theorem 5.2. Suppose to the contrary that $\gamma<\frac{\beta}{2-\alpha}$ and for some $D, d>0$ there are polynomials $P_{n}$ with the properties (i) and (ii) in Theorem 
5.1 for infinitely many n. Let $\tilde{\alpha}<\alpha$ be such that $\gamma<\frac{\beta}{2-\tilde{\alpha}}$ still holds. Then, since the angle of $K$ at $z_{0}$ is $\alpha$, there is an isosceles triangle $\Delta$ lying in $K$ with vertex at $z_{0}$ and of vertex angle $\tilde{\alpha} \pi$. We may assume that $\Delta$ is so small that if we enlarge it from $z_{0}$ by a factor 2 , then it still lies in $K$.

Let $\Delta_{\delta}$ be the translate of $\Delta$ inside $K$ by an amount $\delta$ in the direction of its altitude (corresponding to the vertex at $z_{0}$ ). According to (ii)

$$
\left\|P_{n}\right\|_{\Delta_{\delta}} \leq D e^{-d n^{\beta} \delta^{\gamma}}
$$

So, by the Bernstein-Walsh lemma (6.2), we get that

$$
1=\left|P_{n}(0)\right| \leq D e^{-d n^{\beta} \delta^{\gamma}} e^{n g}{\overline{\mathbf{C} \backslash \Delta_{\delta}}}(0) .
$$

Similarly to $(6.16)$ there is a constant $d_{1}$ such that

$$
g_{\overline{\mathbf{C} \backslash \Delta_{\delta}}}(0) \leq d_{1} \delta^{\frac{1}{2-\widetilde{\alpha}}} .
$$

So we get from $(6.32)$

$$
1 \leq \exp \left(\log D+d_{1} n \delta^{\frac{1}{2-\tilde{\alpha}}}-d n^{\beta} \delta^{\gamma}\right) .
$$

Considering that $\gamma(2-\tilde{\alpha})<\beta$, the exponent on the right-hand side tends to $-\infty$ if $\delta=\delta_{n}=\frac{1}{n^{2-\alpha}}$, which contradicts (6.34).

This contradiction proves Theorem 5.2.

\section{Proof of Theorem 5.3, Remark 5.4 and Exam- ple 5.5}

Proof of Theorem 5.3, (a). Let, as in (a), $\beta<1$.

Without loss of generality we may assume that $z_{0}=0$. Place $K$ so that $y= \pm \tan (\alpha \pi / 2) t, t \geq 0$, are the two half-tangents to $K$ at 0 . If $a$ is small, then, by assumption, the intersection of $K$ with the rectangle

$$
[-2 a, 2 a] \times[-2 a \tan (\alpha \pi / 2), 2 a \tan (\alpha \pi / 2)]
$$

are two $C^{1+\varepsilon}$ smooth arcs $\Gamma_{1}, \Gamma_{2}$ (for some $\varepsilon>0$ ) which are the graphs of some functions

$$
\pm \tan (\alpha \pi / 2) x \pm \gamma_{j}(x), \quad x \in[0, a], j=1,2
$$

where $\gamma_{j}$ are differentiable, their derivative satisfy a Lip $\varepsilon$ condition and $\gamma^{\prime}(0)=$ 0. In particular, $\left|\gamma_{j}(x)\right| \leq C x^{1+\varepsilon}$ for all $x \in[0, a]$ with some constant $C$.

For some small $a$ consider the triangle $\Delta$ enclosed by these two half-tangents $y= \pm \tan (\alpha \pi / 2) x, x \geq 0$ and the line $x=2 a$. Lemma 6.6 yields for this $\Delta$ polynomials $S_{n}$ of degree $\leq C_{0} n$ such that $S_{n}(0)=1$ and

$$
\left|S_{n}(w)\right| \leq 1, \quad\left|S_{n}(w)\right| \leq C_{0} \exp \left(-\left(n|w|^{\frac{1}{2-\alpha}}\right)^{\beta}\right), \quad w \in \Delta
$$


According to what has been said, if $z \in K$ lies sufficiently close to 0 , then the distance from $z$ to $\Delta$ is at most $C_{1}|z|^{1+\varepsilon}$ with some fixed $C_{1}$.

We shall need a test triangle $H$, which we choose as follows. Let $\alpha^{\prime}<\alpha$ be sufficiently close to $\alpha$, and let $H$ be the triangle with vertices $0,\left(a, \pm a \tan \left(\alpha^{\prime} / 2\right)\right)$. (Note: in this part of the proof we could have chosen $\alpha^{\prime}=\alpha$, but in the next proof in the consideration below the role of $K$ and $\Delta$ will have to be reversed, and then $\alpha^{\prime}<\alpha$ may be necessary). If $z_{0}$ is any point on the complex plane then let $H_{z_{0}}=z_{0}+H$ be the translate of $H$ by $z_{0}$. Since the angle $\alpha^{\prime} \pi$ of $H$ at the origin is smaller than $\alpha \pi$ (the angle of $\Delta$ at 0 ), it follows that if $z_{0} \in \Delta$ lies sufficiently close to 0 , then the triangle $H_{z_{0}}$ lies within $\Delta$.

Let $z \in K \backslash \Delta$ lie close to the origin, and let $z_{0} \in \Delta$ be the closest point to $z$ on the horizontal line through $z$. Since the distance from $z$ to $\Delta$ is at most $C_{1}|z|^{1+\varepsilon}$, it follows that $\left|z-z_{0}\right| \leq C_{2}|z|^{1+\varepsilon}$ with some $C_{2}$ that depends only on $\Delta$ and $C_{1}$. Now every point of $H_{z_{0}}$ is of distance $\geq c_{1}|z|$ from the origin (with some $c_{1}>0$ that depends only on $\alpha$ ), hence we obtain from (7.1) the inequality (note that the triangle $H_{z_{0}}$ lies within $\Delta$ )

$$
\left\|S_{n}\right\|_{H_{z_{0}}} \leq C_{0} \exp \left(-\left(n\left(c_{1}|z|\right)^{\frac{1}{2-\alpha}}\right)^{\beta}\right) .
$$

Hence,

$$
\left|S_{n}(z)\right| \leq C_{0} \exp \left(-\left(n\left(c_{1}|z|\right)^{\frac{1}{2-\alpha}}\right)^{\beta}\right) e^{n g_{\overline{\mathbf{C}} \backslash H_{z_{0}}}(z)}
$$

by the Bernstein-Walsh lemma (6.2). Here

$$
g_{\overline{\mathbf{C} \backslash H_{z_{0}}}}(z)=g_{\overline{\mathbf{C} \backslash H}}\left(z-z_{0}\right),
$$

and $z-z_{0}$ is a negative real number. Therefore, (6.8) yields the bound

$$
g_{\overline{\mathbf{C} \backslash H_{z_{0}}}}(z) \leq C_{3}\left|z-z_{0}\right|^{\frac{1}{2-\alpha^{\prime}}} \leq C_{3}\left(C_{2}|z|^{1+\varepsilon}\right)^{\frac{1}{2-\alpha^{\prime}}},
$$

with some $C_{3}$. As a consequence, for another constant $C_{4}$ we have

$$
g_{\overline{\mathbf{C} \backslash H_{z_{0}}}}(z) \leq C_{4}|z|^{\frac{1}{2-\alpha}+\varepsilon^{\prime}},
$$

with some $\varepsilon^{\prime}>0$ provided $\alpha^{\prime}<\alpha$ is so close to $\alpha$ that the inequality $(1+\varepsilon) /(2-$ $\left.\alpha^{\prime}\right)>1 /(2-\alpha)$ is true.

Thus, together with (7.2) we also have

$$
\left|S_{n}(z)\right| \leq C_{0} \exp \left(-\left(n\left(c_{1}|z|\right)^{\frac{1}{2-\alpha}}\right)^{\beta}\right) \exp \left(C_{4} n|z|^{\frac{1}{2-\alpha}+\varepsilon^{\prime}}\right),
$$

and this estimate holds true for all $z \in K$ lying in a neighborhood of the origin.

Let now $\tilde{\Delta}$ be a triangle with vertex at 0 and of the same height as the height of $\Delta$, but its inner angle at 0 let $\tilde{\alpha} \pi$ with an $\tilde{\alpha}>\alpha$ which satisfies

$$
\frac{1}{2-\alpha}+\varepsilon^{\prime}>\frac{1}{2-\tilde{\alpha}}
$$


According to the $\beta=1$ case of Theorem 5.1 there are polynomials $T_{n}$ of degree at most $C_{5} n$ for some $C_{5}$ such that $T_{n}(0)=1$ and on $\tilde{\Delta}$ we have

$$
\left|T_{n}(z)\right| \leq C_{5} \exp \left(-C_{4} n|z|^{\frac{1}{2-\alpha}+\varepsilon^{\prime}}\right) .
$$

Hence, $S_{n} T_{n}$ is a polynomial of degree at most $C_{0} C_{5} n$ which is 1 at the origin and which satisfies

$$
\left|S_{n}(z) T_{n}(z)\right| \leq C_{0} C_{5} \exp \left(-\left(n\left(c_{1}|z|\right)^{\frac{1}{2-\alpha}}\right)^{\beta}\right)
$$

for all $z \in K \cap \tilde{\Delta}$ that lies sufficiently close to 0 . Since this latter set contains a $K$-neighborhood of 0 , on multiplying this $S_{n}(z) T_{n}(z)$ by a sufficiently high degree polynomial from Lemma 6.8 as in the proof of Theorem 5.1 we get polynomials that satisfy properties (i), (ii) (more precisely we shall get a polynomial of degree $\leq C_{6} n$ that satisfies (i), (ii) with $d=c_{1}^{\beta /(2-\alpha)}$, but then these polynomials for $n / C_{6}$ rather than for $n$ are suitable in Theorem 5.3 , (a)).

We still need to verify that the so constructed polynomials satisfy also property (iii), i.e. they are bounded by 1 in absolute value on the set $K$. In view of (7.3) this is certainly the case if $|z| \geq C_{6} / n^{(2-\alpha)}$ with a sufficiently large $C_{6}$, so consider only the case $|z| \leq C_{6} / n^{(2-\alpha)}, z \in K$ (note also that the polynomials $R$ that need to be used as in the proof of Theorem 6.1 and which are guaranteed by Lemma 6.8 are $\leq 1$ in absolute value on $K$ ). All such $z$ belong (for sufficiently large $n$ ) to the triangle $\tilde{\Delta}$, so $\left|T_{n}(z)\right| \leq 1$ is true for them. On the other hand, by the construction in Lemma 6.6 we have for $S_{n}$ the representation

$$
S_{n}(z)=\prod_{k=1}^{m} \frac{Q_{k}(z)}{Q_{k}(0)}
$$

with the polynomials $Q_{k}$ from the proof of Lemma 6.6, and here each of the factors satisfy $\left|Q_{k}(z) / Q_{k}(0)\right| \leq 1$ by Remark 6.7 made after the proof of Lemma 6.6. Hence, $\left|S_{n}(z)\right| \leq 1$, and property (iii) follows.

Proof of Theorem 5.3, (b). Let now $\beta=1$.

In the next section we are going to prove

Theorem 7.1 If $K$ is a triangle with inner angle $\alpha \pi$ at $z_{0}$, then for $\beta=1$ and $\gamma=\frac{1}{2-\alpha}$ and for any $D, d>0$ there is an $n_{0}$ such that for $n \geq n_{0}$ there are no polynomials $P_{n}$ of degree $n$ with the properties (i) and (ii) from Theorem 5.1.

Now suppose that the claim is not true and there are polynomials $P_{n}$ of arbitrary high degree $n$ for which (i) and (ii) in Theorem 5.1 are true for $K$. Place the small triangle $\Delta$ to $K$ as in the just given proof of Theorem 5.3, (a). If we reverse in that proof the role of $K$ and $\Delta$ then we obtain that for $\Delta$ there are polynomials $P_{n}$ of arbitrary high degree for which (i) and (ii) are true, but this is not the case according to Theorem 7.1. We omit the details. 
Proof of Remark 5.4. The remark claims the following:

(a) If $\beta<1$, then for $\gamma=\beta$ there are polynomials $P_{n}$ with properties (i)-(iii).

(b) If $\beta=1$, then for $\gamma>1$ there are polynomials $P_{n}$ with properties (i)-(iii).

(c) If $\beta=1$ and $\gamma=1$, then for any $D, d>0$ there is an $n_{0}$ such that for $n \geq n_{0}$ there are no polynomials $P_{n}$ of degree $n$ with the properties (i) and (ii).

(d) For any $\beta \leq 1$ and $\gamma<\beta$, then for any $D, d>0$ there is an $n_{0}$ such that for $n \geq n_{0}$ there are no polynomials $P_{n}$ of degree $n$ with the properties (i) and (ii).

The existence parts in (a) and (b) were proven in [25, Theorem 4.1] and in [26, Lemma 4] (in those results only the existence of the disk in the complement containing $z_{0}$ on its boundary was used).

As for part (c), consider the proof of Theorem 5.3. Suppose that the position of $K$ is similar as in that proof, namely the positive and negative parts of the imaginary axis are the two half tangents to $K$ at $z_{0}=0$, and $K$ contains a small interval $\left[0, a_{0}\right]$. Replace in those proofs the triangle $\Delta$ by a square $[0,2 a] \times[-a, a]$. Now if there were polynomials as in (c) for infinitely many $n$ then the proof given for Theorem 5.3 would give polynomials $H_{n}$ of degree at most $n$ such that $H_{n}(0)=1$, and $\left|H_{n}(i t)\right| \leq D_{1} e^{-n d_{1}|t|}$ for all $t \in[-a, a]$, which is impossible by [10, Theorem 1] (see also [10, Corollary 4]).

Finally, for the proof of (d) just follow the proof of Theorem 5.2.

The construction in Example 5.5. Consider the function

$$
\varphi(t)=\left(\tan \frac{\alpha \pi}{2}\right) t+\int_{0}^{t} \frac{1}{\log |\log u|} d u
$$

on the interval $\left[0,10^{-2}\right]$, and let $K$ be bounded by the graph of $\pm \varphi$ and of the segment connecting the points $\left(10^{-2},-\varphi\left(10^{-2}\right)\right)$ and $\left(10^{-2}, \varphi\left(10^{-2}\right)\right)$. Clearly, the angle of $K$ at 0 is $\alpha \pi$, and the boundary of $K$ is piecewise $C^{1}$ smooth. For $\tau \in\left(0,10^{-2} / 2\right)$ the tangent line to the curve of $\varphi$ at the point $(\tau, \varphi(\tau))$ has slope

$$
\varphi^{\prime}(\tau)=\tan \frac{\alpha \pi}{2}+\frac{1}{\log |\log \tau|},
$$

and that tangent line intersects the $x$ axis at the point $(\delta, 0)$ with

$$
\begin{aligned}
\delta & =\delta(\tau):=\frac{\tan (\alpha \pi / 2) \tau+\tau / \log |\log \tau|-\varphi(\tau)}{\varphi^{\prime}(\tau)} \\
& =\frac{\tan (\alpha \pi / 2) \tau+\tau / \log |\log \tau|-\varphi(\tau)}{\tan (\alpha \pi / 2)+1 / \log |\log \tau|} \leq \tau .
\end{aligned}
$$


If $\frac{\tilde{\alpha} \pi}{2}$ is the angle between the $x$ axis and that tangent line then

$$
\tan \frac{\tilde{\alpha} \pi}{2}=\tan \frac{\alpha \pi}{2}+\frac{1}{\log |\log \tau|},
$$

from which it follows that

$$
\tilde{\alpha}-\alpha \sim \frac{1}{\log |\log \tau|} .
$$

Note that from the construction it follows that the isosceles triangle $\Delta_{\delta}$ with vertex at $\delta$, of vertex angle $\tilde{\alpha} \pi$ and of altitude $10^{-3}$ is part of $K$, hence if there are polynomials $P_{n}$ for infinitely many $n$ with properties (i) and (ii) in Theorem 5.1 , then we can apply formula (6.31) with $\gamma=\frac{\beta}{2-\alpha}$ :

$$
\left\|P_{n}\right\|_{\Delta_{\delta}} \leq D e^{-d n^{\beta} \delta^{\gamma}}
$$

and then exactly as in (6.32)

$$
1=\left|P_{n}(0)\right| \leq D e^{-d n^{\beta} \delta^{\gamma}} e^{n g_{\overline{\mathbf{C}} \backslash \Delta_{\delta}}(0)} .
$$

Now the estimate (6.33) holds uniformly in $\tilde{\alpha}$ lying in a closed subinterval of $(0,1)$, hence there is a constant $d_{1}$ such that

$$
g_{\overline{\mathbf{C} \backslash \Delta_{\delta}}}(0) \leq d_{1} \delta^{\frac{1}{2-\tilde{\alpha}}},
$$

and so we obtain from $(7.5)$

$$
1 \leq \exp \left(\log D+d_{1} n \delta^{\frac{1}{2-\widetilde{\alpha}}}-d n^{\beta} \delta^{\gamma}\right) .
$$

For large $n$ choose $\tau$ so that for the resulting $\delta=\delta(\tau)$ we have $n \delta^{\frac{1}{2-\bar{\alpha}}}=1$, i.e. $\delta=1 / n^{2-\tilde{\alpha}}$ (but note that $\tilde{\alpha}$ also depends on $\tau$ ). Since $\gamma=\beta /(2-\alpha)$, the last term in the exponent in (7.7) is then

$$
-d\left(n^{1-\frac{2-\tilde{\alpha}}{2-\alpha}}\right)^{\beta}=-d\left(n^{\frac{\tilde{\alpha}-\alpha}{2-\alpha}}\right)^{\beta} .
$$

Since $\tau \geq \delta=1 / n^{2-\tilde{\alpha}}$ and $\tilde{\alpha}-\alpha \sim 1 / \log |\log \tau|$, we infer the existence of a constant $d_{2}$ independent of $n$ such that $\tilde{\alpha}-\alpha \geq d_{2} / \log |\log n|$, and

$$
n^{\frac{\tilde{\alpha}-\alpha}{2-\alpha}} \geq e^{c \log n / \log \log n},
$$

so the exponent on the right-hand side of (7.7) tends to $-\infty$, which is a contradiction. This contradiction shows that for the $K$ above there are no polynomials $P_{n}$ (for infinitely many $n$ ) that would satisfy (i) and (ii) in Theorem 5.1. 


\section{Proof of Theorem 7.1}

For $s>0$ and $0<\alpha<1$ let $\tau=e^{i \alpha \pi / 2}, \Sigma^{+}$the segment connecting 0 with $\tau s$, $\Sigma^{-}$the segment connecting 0 with $\bar{\tau} s$ and let $\Sigma=\Sigma^{+} \cup \Sigma^{-}$be the wedge from $s e^{-i \alpha \pi / 2}$ to 0 and then to $s e^{i \alpha \pi / 2}$. This $\Sigma$ has two sides, we consider the side of $\Sigma$ pointing towards the negative real axis its negative side (denoted by $\Sigma_{-}$) and the side pointing towards the positive real axis its positive side (denoted by $\left.\Sigma_{+}\right)$. Then at the origin $\Sigma_{+}$has an angle $\alpha \pi$, while $\Sigma_{-}$has an angle $(2-\alpha) \pi$.

Theorem 7.1 is clearly a consequence of

Theorem 8.1 Let $s>0$ and $0<\alpha<1$ be arbitrary. Then for any $D, d>0$ there is an $n_{0}$ such that for $n \geq n_{0}$ there are no polynomials $P_{n}$ of degree $n$ with the properties

(i) $P_{n}(0)=1$,

(ii) $\left|P_{n}(z)\right| \leq D \exp \left(-d n|z|^{\frac{1}{2-\alpha}}\right), \quad z \in \Sigma$.

Proof. For the proof we need some results from the theory of weighted potentials, see the book [21].

On $\Sigma$ consider the "external field" $Q(z)=-(d / 2)|z|^{\frac{1}{2-\alpha}}$ and the weight function $w(z)=\exp (-Q(z))=\exp \left((d / 2)|z|^{\frac{1}{2-\alpha}}\right)$. There is a unique probability Borel measure $\mu_{w}$ on $\Sigma$ that minimizes the weighted energy

$$
\iint \log \frac{1}{|z-t|} d \mu(t) d \mu(z)+2 \int Q(t) d \mu(t)
$$

(see [21, Theorem I.1.3]). Let $\mathcal{S}_{w}$ denote the support of $\mu_{w}$.

The proof will proceed as follows. Assume to the contrary that polynomials $P_{n}$ with properties (i)-(ii) exist for arbitrary large degrees. We are going to show that then

- $\mathcal{S}_{w}=\Sigma$,

- $\mu_{w}$ is absolutely continuous on $\Sigma$ with respect to arc (linear) measure on $\Sigma$, and its density is obtained from the logarithmic potential of $\mu_{w}$ by taking normal derivatives,

- this density formula produces a negative function close to the origin.

Since the density of a positive measure has to be nonnegative, this latter fact will establish the required contradiction.

In this argument central role is played by the logarithmic potential

$$
U(z)=\int \log \frac{1}{|z-t|} d \mu_{w}(t) .
$$

In our case for $U$ we know that it is continuous everywhere ([21, Theorem I.5.1(iv)']), and there is a constant $F$ such that (see [21, Theorem I.1.3])

$$
U(z)-(d / 2)|z|^{\frac{1}{2-\alpha}} \leq F, \quad z \in \mathcal{S}_{w}
$$


and

$$
U(z)-(d / 2)|z|^{\frac{1}{2-\alpha}} \geq F, \quad \text { for } z \in \Sigma, \text { except for a set of zero capacity. }
$$

Since $U$ is continuous, there is actually no exceptional set in the latter inequality, i.e.

$$
U(z)-(d / 2)|z|^{\frac{1}{2-\alpha}} \geq F, \quad z \in \Sigma .
$$

In particular,

$$
U(z)-(d / 2)|z|^{\frac{1}{2-\alpha}}=F, \quad z \in \mathcal{S}_{w} .
$$

The unicity of $\mu_{w}$ and the symmetry of $\Sigma$ onto the real line implies that $\mu_{w}$ is symmetric onto the real line. In particular, $\mathcal{S}_{w}$ is also symmetric onto $\mathbf{R}$, hence

$$
\begin{aligned}
U(\bar{\tau} x) & =\int_{0}^{s}\left(\log \frac{1}{|\bar{\tau} x-\bar{\tau} t|}+\log \frac{1}{|\bar{\tau} x-\tau t|}\right) d \mu_{w}(\bar{\tau} t) \\
& =\int_{0}^{s}\left(\log \frac{1}{|x-t|}+\log \frac{1}{\left|x-\tau^{2} t\right|}\right) d \mu_{w}(\bar{\tau} t) .
\end{aligned}
$$

First we claim that $\Sigma^{-} \cap \mathcal{S}_{w}$ is a segment. Indeed, suppose this is not the case. Then there are $0<a<b$ such that $\bar{\tau} a, \bar{\tau} b \in \mathcal{S}_{w}$, but no $\bar{\tau} t$ lies in $\mathcal{S}_{w}$ for $t \in(a, b)$. For a fixed $t \in[0,1] \backslash(a, b)$ consider the second derivative of

$$
\log \frac{1}{|x-t|}+\log \frac{1}{\left|x-\tau^{2} t\right|}, \quad \tau^{2}=e^{i \alpha \pi},
$$

with respect to $x$ on the interval $(a, b)$. It is

$$
\frac{1}{(x-t)^{2}}-\frac{1}{x^{2}+t^{2}-2 x t \cos \alpha \pi}+\frac{2(x-t \cos \alpha \pi)^{2}}{\left(x^{2}+t^{2}-2 x t \cos \alpha \pi\right)^{2}},
$$

which is clearly positive (the absolute value of the second term is smaller than the first term). Thus, in view of (8.3), the function $U(\bar{\tau} x)$ is strictly convex on $[a, b]$. Since so is $-(d / 2)|\bar{\tau} x|^{\frac{1}{2-\alpha}}$, we get a contradiction to (8.1) and (8.2), because $U(\bar{\tau} x)-(d / 2)|\bar{\tau} x|^{\frac{1}{2-\alpha}}$ is less than $F$ on $(a, b)$ since it is convex and it equals $F$ at the two endpoints $a$ and $b$. This contradiction proves that, indeed, $\Sigma^{-} \cap \mathcal{S}_{w}$ is a segment (and by symmetry, the same is true of $\Sigma^{+} \cap \mathcal{S}_{w}$ ).

Assume now to the contrary that there are $D, d>0$ and infinitely many $n$ and $P_{n}$ such that (i) and (ii) in Theorem 8.1 are true. We claim that in this case $\mathcal{S}_{w}$ is the whole $\Sigma$. To this end we need the following characterization of the support $\mathcal{S}_{w}$ of $\mu_{w}$ ([21, Theorem IV.1.3]): $z_{0} \in \mathcal{S}_{w}$ if and only if for any $\delta>0$ there is a polynomial $S_{n}$ of degree $n$ such that $w^{n}(z)\left|S_{n}(z)\right|=$ $\exp \left(n(d / 2)|\tau x|^{\frac{1}{2-\alpha}}\right)\left|S_{n}(z)\right|$ attains its maximum on $\Sigma$ in the neighborhood $\mid z-$ $z_{0} \mid<\delta$ and nowhere else. Since (ii) implies

$$
w^{n}(z)\left|P_{n}(z)\right|=\exp \left(n(d / 2)|z|^{\frac{1}{2-\alpha}}\right)\left|P_{n}(z)\right| \leq D \exp \left(-n(d / 2)|z|^{\frac{1}{2-\alpha}}\right),
$$


this is certainly the case with $z_{0}=0$, so $0 \in \mathcal{S}_{w}$. On the other hand, the polynomial $Q_{1}(z)=z-\tau s / 2$ assumes its absolute maximum on $\Sigma$ at the endpoint $\bar{\tau} s \in$ $\Sigma^{-}$, and hence the same is true of $w(z)\left|Q_{1}(z)\right|=\exp \left((d / 2)|z|^{\frac{1}{2-\alpha}}\right)|z-\tau s / 2|$, so $\bar{\tau} s$ also lies in $\mathcal{S}_{w}$. Since $\Sigma^{-} \cap \mathcal{S}_{w}$ is a segment, it follows that $\Sigma^{-} \cap \mathcal{S}_{w}=\Sigma^{-}$, and from symmetry we can conclude the claim that $\mathcal{S}_{w}=\Sigma$.

Let $\Omega=\overline{\mathbf{C}} \backslash \Sigma$ be the complement of $\Sigma$, and $g_{\Omega}(z)$ the Green's function of $\Omega$ with pole at infinity. The function $U(z)+g_{\Omega}(z)$ is continuous on the whole plane, it is harmonic outside $\Sigma$ (including $\infty$ ) and it equals $F+(d / 2)|z|^{\frac{1}{2-\alpha}}$ on $\Sigma$ (see (8.1)-(8.2)), hence it is the solution of the Dirichlet problem with that boundary function. Let $h(z)$ be the solution of the Dirichlet problem in $\Omega$ with boundary function $|z|^{\frac{1}{2-\alpha}}$ on $\Sigma$. Thus,

$$
U(z)=(d / 2) h(z)-g_{\Omega}(z)+F, \quad z \in \mathbf{C} .
$$

It is standard that on any closed subsegment of $\Sigma$ that does not contain either of the points $0, s \tau, s \bar{\tau}$, both $g_{\Omega}$ and $h$ (considered as functions on $\mathbf{C}$ ) are Lip 1 functions (see the Remark after the proof). Hence, $U$ is a Lip 1 function away from the points $0, s \tau, s \bar{\tau}$, so we can apply [21, Theorem II.1.5] to conclude from $\mathcal{S}_{w}=\Sigma$ that $\mu_{w}$ is absolutely continuous on $\Sigma$ with respect to arc length, and if $\omega(z)$ denotes its density then

$$
\omega(z)=-\frac{1}{2 \pi}\left(\frac{\partial U(z)}{\partial \mathbf{n}_{+}}+\frac{\partial U(z)}{\partial \mathbf{n}_{-}}\right),
$$

where $\mathbf{n}_{ \pm}$denote the normals to $\Sigma$ at $z \in \Sigma$ towards the positive and negative sides of $\Sigma$. What we are going to show below is that the right-side of (8.5) is negative if $z \in \Sigma$ is sufficiently close to 0 , and this contradiction proves the claim in the theorem (since we obtained the contradiction from the assumption that there are polynomials $P_{n}$ with properties (i)-(ii)).

Thus, our task is to analyze the normal derivatives in (8.5) and to prove that for $z \in \Sigma$ lying close the origin the right-side of (8.5) is negative. In view of (8.4) we shall deal with the normal derivatives of $h$ and $g_{\Omega}(z)$ separately.

Let $\varphi$ be the conformal map from the unit disk $D$ onto $\Omega=\overline{\mathbf{C}} \backslash \Sigma$ which maps 0 into $\infty$ and 1 into $0 \in \Sigma_{-}$. Since $\overline{\varphi(\bar{z})}$ also has these properties, the unicity of $\varphi$ gives that $\varphi(z)=\overline{\varphi(\bar{z})}$. In particular, $\varphi(-1)=0\left(\in \Sigma_{+}\right)$, the upper half of the unit circle $C_{1}$ is mapped onto $\Sigma^{+}$and the lower half is mapped onto $\Sigma^{-}$. At $\zeta=1$ the boundary $\varphi\left(C_{1}\right)$ has an angle $\theta=(2-\alpha) \pi$, while at $\zeta=-1$ the boundary $\varphi\left(C_{1}\right)$ has an angle $\alpha \pi$. The two arcs forming those angles are straight segments, i.e. analytic arcs, hence by Lehman's theorem [13], [19, Sec. 3.4, p.58], in the unit disk $\varphi$ has the following expansion close to $\zeta= \pm 1$ (with the corresponding $\theta=2-\alpha$ or $\alpha$ and with appropriate branches of $(z-\zeta)^{\theta}$ and $\log (z-\zeta))$. If $\theta$ is irrational, then

$$
\begin{aligned}
\varphi(z) & =\sum_{k=0}^{\infty} \sum_{j=1}^{\infty} a_{k, j}(z-\zeta)^{k+\theta j} \\
& =a_{01}(z-\zeta)^{\theta}\left(1+\frac{a_{02}}{a_{01}}(z-\zeta)^{\theta}+\frac{a_{11}}{a_{01}}(z-\zeta)+\cdots\right), \quad a_{01} \neq 0,
\end{aligned}
$$


while if $\theta=p / q$ is rational with relative prime $p, q$, then with some $a_{010} \neq 0$

$$
\begin{aligned}
\varphi(z) & =\sum_{k=0}^{\infty} \sum_{j=1}^{\infty} \sum_{m=0}^{[k / p]} a_{k j m}(z-\zeta)^{k+\theta j}(\log (z-\zeta))^{m}=a_{010}(z-\zeta)^{\theta} \times \\
& \times\left(1+\frac{a_{020}}{a_{010}}(z-\zeta)^{\theta}+\frac{a_{11[1 / p]}}{a_{010}}(z-\zeta)(\log (z-\zeta))^{[1 / p]}+\cdots\right) .
\end{aligned}
$$

These imply (we skip the computation) that if we set

$$
f(x)=\left|\varphi\left(e^{i x}\right)\right|^{\frac{1}{2-\alpha}},
$$

then in the case $\zeta=-1, \theta=\alpha$, we have with $\eta:=\alpha /(2-\alpha)$ and with some constant $C^{1}$

$$
0 \leq f(x+\pi) \leq C|x|^{\eta}, \quad\left|f^{\prime \prime}(x+\pi)\right| \leq C|x|^{\eta-2}, \quad|x| \leq 1,
$$

while at $\zeta=1$, i.e. when $\theta=2-\alpha>1$, we have that

$$
f(x)=c_{1}|x|+f_{0}(x)
$$

with some constant $c_{1}>0$ and with an $f_{0}$ satisfying

$$
\left|f_{0}(x)\right| \leq C|x|^{2}, \quad\left|f_{0}^{\prime \prime}(x)\right| \leq C, \quad|x| \leq 1 .
$$

Let

$$
H\left(r e^{i x}\right)=\frac{1}{2 \pi} \int_{-\pi}^{\pi} \frac{f(t)\left(1-r^{2}\right)}{1-2 r \cos (x-t)+r^{2}} d t
$$

be the Poisson integral of $f$. Since this has boundary value

$$
f(x)=\left|\varphi\left(e^{i x}\right)\right|^{\frac{1}{2-\alpha}}=h\left(\varphi\left(e^{i x}\right)\right)
$$

at $e^{i x}$, it follows that $H(z)=h(\varphi(z))$. If $\mathbf{n}$ denotes inner normal at $e^{i x}$ to the unit circle, then (in a neighborhood of the origin) one can easily see the transformation formula (cf. (4.12))

$$
\frac{\partial h(z)}{\partial \mathbf{n}_{-}}=\frac{\partial H\left(e^{i x}\right)}{\partial \mathbf{n}} \frac{1}{\left|\varphi^{\prime}\left(e^{i x}\right)\right|}, \quad z=\varphi\left(e^{i x}\right),
$$

while for the normal derivative in the other direction we have

$$
\frac{\partial h(z)}{\partial \mathbf{n}_{+}}=\frac{\partial H\left(-e^{i x}\right)}{\partial \mathbf{n}} \frac{1}{\left|\varphi^{\prime}\left(-e^{i x}\right)\right|}, \quad z=\varphi\left(-e^{i x}\right) .
$$

In (8.10) and (8.11) $x$ is considered to be sufficiently close to 0 (so that $\varphi\left( \pm e^{i x}\right)$ lies on the side $\Sigma_{\mp}$ ).

\footnotetext{
${ }^{1}$ The constant $C$ below may change from one place to the next one even within a line.
} 
Consider first (8.11). In that case $\zeta=-1, \eta=\alpha /(2-\alpha), \theta=\alpha$ and (in a neighborhood of the origin) $\left|\varphi\left(-e^{i x}\right)\right| \sim|x|^{\alpha},\left|\varphi^{\prime}\left(-e^{i x}\right)\right| \sim|x|^{\alpha-1}$ (meaning that the ratio of the two sides lies in between two constants), hence from (8.6) and from Lemma 8.3 below we can conclude that

$$
\left|\frac{\partial h(z)}{\partial \mathbf{n}_{+}}\right| \leq C|x|^{\eta-1} \frac{1}{|x|^{\alpha-1}}=C|x|^{\frac{\alpha}{(2-\alpha)}-\alpha} \leq C|z|^{\frac{1}{2-\alpha}-1}, \quad z=\varphi\left(-e^{i x}\right) .
$$

The decomposition (8.7) yields the decomposition $H(z)=H_{0}(z)+c_{1} H_{1}(z)$, where $H_{0}$ is the Poisson integral of $f_{0}$ and $H_{1}(z)$ is the Poisson integral of $f_{1}(x)=|x|, x \in[-\pi, \pi]$. In (8.10) we have $\zeta=1, \theta=2-\alpha,\left|\varphi\left(e^{i x}\right)\right| \sim$ $|x|^{2-\alpha},\left|\varphi^{\prime}\left(e^{i x}\right)\right| \sim|x|^{2-\alpha-1}$, hence from (8.8) and from Lemma 8.3 below we can conclude that

$$
\left|\frac{\partial H_{0}\left(e^{i x}\right)}{\partial \mathbf{n}}\right| \frac{1}{\left|\varphi^{\prime}\left(e^{i x}\right)\right|} \leq C \frac{1}{|x|^{2-\alpha-1}} \leq C|z|^{\frac{1}{2-\alpha}-1}, \quad z=\varphi\left(e^{i x}\right) .
$$

On the other hand, Lemma 8.4 shows that for $x$ lying close to 0

$$
\frac{\partial H_{1}\left(e^{i x}\right)}{\partial \mathbf{n}} \frac{1}{\left|\varphi^{\prime}\left(e^{i x}\right)\right|} \geq c_{2} \frac{1}{|x|^{2-\alpha-1}} \log \frac{1}{|x|} \geq c_{3}|z|^{\frac{1}{2-\alpha}-1} \log \frac{1}{|z|}, \quad z=\varphi\left(e^{i x}\right),
$$

with some $c_{3}>0$. These last two inequalities prove (see also (8.10)) that

$$
\frac{\partial h(z)}{\partial \mathbf{n}_{-}}=\frac{\partial H_{0}\left(e^{i x}\right)}{\partial \mathbf{n}} \frac{1}{\left|\varphi^{\prime}\left(e^{i x}\right)\right|}+c_{1} \frac{\partial H_{1}\left(e^{i x}\right)}{\partial \mathbf{n}} \frac{1}{\left|\varphi^{\prime}\left(e^{i x}\right)\right|} \geq c_{4}|z|^{\frac{1}{2-\alpha}-1} \log \frac{1}{|z|} .
$$

Next, let $G(z)=g_{\Omega}(\varphi(z))$. This is the Green's function in the unit disk with pole at the origin, so $G(z)=\log \frac{1}{|z|}$. This has normal derivative 1 at every point of the unit circle, so we get from the analogues of (8.10) and (8.11) (replace $h, H$ with $g, G$ there) that

$$
\left|\frac{\partial g(z)}{\partial \mathbf{n}_{+}}\right| \leq C \frac{1}{\left|\varphi^{\prime}\left(-e^{i x}\right)\right|} \leq C \frac{1}{|x|^{\alpha-1}} \leq C|z|^{\frac{1}{\alpha}-1}, \quad z=\varphi\left(-e^{i x}\right),
$$

and

$$
\left|\frac{\partial g(z)}{\partial \mathbf{n}_{-}}\right| \leq C \frac{1}{\left|\varphi^{\prime}\left(e^{i x}\right)\right|} \leq C \frac{1}{|x|^{(2-\alpha)-1}} \leq C|z|^{\frac{1}{2-\alpha}-1}, \quad z=\varphi\left(e^{i x}\right),
$$

in a neighborhood of the origin. Since $1 / \alpha>1>1 /(2-\alpha)$, it follows that

$$
\left|\frac{\partial g(z)}{\partial \mathbf{n}_{ \pm}}\right| \leq C|z|^{\frac{1}{2-\alpha}-1} .
$$

Now (8.12)-(8.14) and the representation (8.4) yield that for $z \in \Sigma, z \neq 0$, lying sufficiently close to the origin the right-hand side of (8.5) is negative, which is what we wanted to prove. 
Remark 8.2 The mapping $\varphi$ is analytic at every point of the unit circle that is not mapped into either of $0, s \tau, s \bar{\tau}$, therefore the above argument and simple properties of the Poisson kernel easily yield that both $g_{\Omega}$ and $h$ are Lip 1 functions (considered as functions) on any closed segment of $\Sigma$ that does not contain either of $0, s \tau, s \bar{\tau}$. This property was used in (8.5).

In the above proof we used the following two lemmas.

Lemma 8.3 Let $f$ be a continuous function on $[-\pi, \pi]$ and let $H(z)$ be its Poisson integral (8.9). Assume that $f$ is twice differentiable on some $(-\varepsilon, 0) \cup$ $(0, \varepsilon)$ and with some $\eta, C>0$ it satisfies

$$
|f(x)| \leq C|x|^{\eta}, \quad\left|f^{\prime \prime}(x)\right| \leq C|x|^{\eta-2}
$$

there.

(a) If $0<\eta<1$, then the normal derivative of $H$ at $e^{i x}$ satisfies the inequality

$$
\left|\frac{\partial H\left(e^{i x}\right)}{\partial \mathbf{n}}\right| \leq C_{1}|x|^{\eta-1}
$$

in a neighborhood of the origin.

(b) If $\eta>1$, then the normal derivative of $H$ at $e^{i x}$ is uniformly bounded in a neighborhood of the origin.

Proof. We may assume $0<x<\varepsilon / 2<1 / 2$. We have

$$
\frac{\partial H\left(e^{i x}\right)}{\partial \mathbf{n}}=\lim _{r \rightarrow 1} \frac{H\left(r e^{i x}\right)-H\left(e^{i x}\right)}{1-r}=\lim _{r \rightarrow 1} \frac{1}{2 \pi} \int_{-\pi}^{\pi} \frac{(f(t)-f(x))(1+r)}{1-2 r \cos (x-t)+r^{2}} d t .
$$

The integral over $|x-t| \geq 1$ is clearly uniformly bounded in $r$, therefore we shall only need to estimate

$$
\lim _{r \rightarrow 1} \int_{x-1}^{x+1} \frac{(f(t)-f(x))(1+r)}{1-2 r \cos (x-t)+r^{2}} d t=\int_{0}^{1} \frac{(f(x-u)+f(x+u)-2 f(x))}{1-\cos u} d u
$$

(that the limit exists follows from the reasonings below and from the bounded convergence theorem). For $0 \leq u \leq x / 2$ we get from the mean value theorem and from the assumptions that the absolute value of the numerator on the right is $\leq C x^{\eta-2} u^{2}$, and for $u \geq x / 2$ it is $\leq C u^{\eta}$. At the same time the denominator is

$$
1-\cos u=2 \sin ^{2} \frac{u}{2} \geq 2\left(\frac{2}{\pi} \frac{u}{2}\right)^{2}
$$

These give the following bound for $(8.16)$ when $\eta<1$ :

$$
\leq C \int_{0}^{x / 2} x^{\eta-2} d u+C \int_{x / 2}^{1} u^{\eta-2} d u \leq C x^{\eta-1} .
$$


In a similar vein, (8.16) is bounded in a neighborhood of the origin if $\eta>1$, which proves part (b).

Lemma 8.4 Let $f(x)=|x|$ for $x \in[-\pi, \pi]$ and let $H(z)$ be its Poisson integral (8.9). In a neighborhood of the origin the normal derivative of $H$ at $e^{i x}$ satisfies the inequality

$$
\frac{\partial H\left(e^{i x}\right)}{\partial \mathbf{n}} \geq c_{0} \log \frac{1}{|x|}
$$

with some $c_{0}>0$.

Proof. Let $0<x<1$. By the preceding proof

$$
\frac{\partial H\left(e^{i x}\right)}{\partial \mathbf{n}}=O(1)+\int_{0}^{1} \frac{|x-u|+|x+u|-2|x|}{1-\cos u} d u .
$$

Here the integrand is 0 for $0 \leq u \leq x$ and it is $2(u-x)$ for $u>x$, therefore

$$
\frac{\partial H\left(e^{i x}\right)}{\partial \mathbf{n}}=O(1)+\int_{x}^{1} \frac{2(u-x)}{1-\cos u} d u \geq O(1)+\int_{2 x}^{1} \frac{u}{1-\cos u} d u,
$$

from which the claim is immediate since $1-\cos u=2 \sin ^{2} \frac{u}{2} \leq u^{2} / 2$.

\section{Nikolskii type inequalities for area measures}

Let $K$ be a compact set with piecewise $C^{1+}$ smooth boundary such that $\overline{\mathbf{C}} \backslash K$ is simply connected (actually, what follows is true in the finitely connected case without much change). For $1 \leq p<\infty$ let

$$
\|f\|_{L^{p}(K)}=\left(\int_{K}|f|^{p}\right)^{1 / p}
$$

be the $L^{p}$-norm with respect to area measure on $K$, and for $p=\infty$ let $\|\cdot\|_{K}=$ $\|\cdot\|_{L^{\infty}(K)}$ be the standard supremum norm. By Hölder's inequality we have for $p \leq q$ the inequality

$$
\|f\|_{L^{p}(K)} \leq C\|f\|_{L^{q}(K)}
$$

with a $C$ that depends only on the area of $K$. For polynomials we have a converse inequality

$$
\left\|P_{n}\right\|_{L^{q}(K)} \leq L\left\|P_{n}\right\|_{L^{p}(K)}
$$

where $L$ depends on $K$, as well as the degree of the polynomial. This latter dependence is heavily influenced by the smallest inner angle at the corners of $K$, as is shown by 
Theorem 9.1 Let $K$ be as before with piecewise $C^{1+}$ smooth boundary, and let $\alpha \pi$ be the smallest internal angle of $K$, which we assume to be positive. Then there is a constant $C$ depending only on $K$ such that for $1 \leq p<q \leq \infty$ and any polynomial $P_{n}$ of degree at most $n=1,2, \ldots$ we have

$$
\left\|P_{n}\right\|_{L^{q}(K)} \leq C n^{2\left(2-\alpha^{*}\right)\left(\frac{1}{p}-\frac{1}{q}\right)}\left\|P_{n}\right\|_{L^{p}(K)},
$$

where $\alpha^{*}=\min (\alpha, 1)$.

This estimate is sharp, for there are nonzero polynomials $P_{n}$ of degree $n=$ $1,2, \ldots$ such that

$$
\left\|P_{n}\right\|_{L^{q}(K)} \geq c n^{2\left(2-\alpha^{*}\right)\left(\frac{1}{p}-\frac{1}{q}\right)}\left\|P_{n}\right\|_{L^{p}(K)}
$$

with some constant $c>0$.

This is the complete analogue of the classical Nikolskii inequality, see e.g. [7, Theorem 4.2.6].

Proof of (9.1). First of all, it is sufficient to prove the $q=\infty$ case. Indeed, suppose we know that

$$
\left\|P_{n}\right\|_{L^{\infty}(K)} \leq C n^{2\left(2-\alpha^{*}\right) \frac{1}{p}}\left\|P_{n}\right\|_{L^{p}(K)} .
$$

Then

$$
\int_{K}\left|P_{n}\right|^{q} \leq \int_{K}\left|P_{n}\right|^{p}\left\|P_{n}\right\|_{L^{\infty}(K)}^{q-p},
$$

and if we apply here (9.3) then we get

$$
\int_{K}\left|P_{n}\right|^{q} \leq \int_{K}\left|P_{n}\right|^{p}\left(C n^{2\left(2-\alpha^{*}\right) \frac{1}{p}}\left\|P_{n}\right\|_{L^{p}(K)}\right)^{q-p},
$$

which is (9.1).

As for the proof of (9.3) let $M=\left\|P_{n}\right\|_{K}$, and let $z_{0} \in K$ be a point such that this maximum is attained: $\left|P_{n}\left(z_{0}\right)\right|=M$. According to (6.6) the Green's function $g_{\overline{\mathbf{C} \backslash K}}$ satisfies the bound

$$
g_{\overline{\mathbf{C} \backslash K}}(w) \leq C_{1}(\operatorname{dist}(w, K))^{\frac{1}{2-\alpha^{*}}}
$$

with some constant $C_{1}$, and since $\left\|P_{n}\right\|_{K}=M$, it follows from the BernsteinWalsh lemma (6.2) that in the $1 / n^{2-\alpha^{*}}$-neighborhood of $K$ we have the bound

$$
\left|P_{n}(w)\right| \leq M e^{C_{1}} .
$$

Use this and Cauchy's formula

$$
P_{n}^{\prime}(z)=\frac{1}{2 \pi i} \int_{|z-\xi|=\delta} \frac{P_{n}(\xi)}{(\xi-z)^{2}} d \xi
$$


with $\delta=1 / n^{2-\alpha^{*}}$ to conclude that on $K$ we have

$$
\left|P_{n}^{\prime}(z)\right| \leq M e^{C_{1}} n^{2-\alpha^{*}} .
$$

Since $\left|P_{n}\left(z_{0}\right)\right|=M$ and (for large $n$ ) any point $w \in K$ which is of distance $\mid w-$ $z_{0} \mid \leq 1 / 4 e^{C_{1}} n^{\left(2-\alpha^{*}\right)}$ from $z_{0}$ can be connected with $z_{0}$ in $K$ by a smooth path of length $\leq 1 / 2 e^{C_{1}} n^{\left(2-\alpha^{*}\right)}$, it follows that at every such $w$ we have $\left|P_{n}(w)\right| \geq M / 2$. Since the set of such $w \in K$ 's has area $\geq c_{1}\left(1 / 4 e^{C_{1}} n^{\left(2-\alpha^{*}\right)}\right)^{2}$ with a $c_{1}$ that depends only on $K$, it follows that

$$
\int_{K}\left|P_{n}\right|^{p} \geq c_{1}\left(\frac{1}{4 e^{C_{1}} n^{2-\alpha^{*}}}\right)^{2}\left(\frac{M}{2}\right)^{p},
$$

which implies (9.3).

Proof of (9.2). Let $V$ be the vertex of $K$ with smallest inner angle $\alpha \pi$, and suppose first that $\alpha<1$ (by the assumption in the theorem this $\alpha$ is positive). Without loss of generality assume that $V=0$, and for this point consider the fast decreasing polynomials $P_{n}$ of Theorem 5.3(a) with $\beta=1 / 2$ and $\gamma=\frac{1}{2(2-\alpha)}$ :

$$
P_{n}(0)=1, \quad\left|P_{n}(z)\right|^{p} \leq D \exp \left(-d p\left(n|z|^{\frac{1}{2-\alpha}}\right)^{1 / 2}\right) .
$$

Since for $k=0,1,2, \ldots$ and

$$
2^{k} n^{-(2-\alpha)} \leq|z| \leq 2^{k+1} n^{-(2-\alpha)}, \quad z \in K,
$$

we have

$$
\left|P_{n}(z)\right|^{p} \leq D \exp \left(-d p\left(n|z|^{\frac{1}{2-\alpha}}\right)^{1 / 2}\right) \leq D e^{-d p 2^{k / 2(2-\alpha)}},
$$

it follows that

$$
\int_{2^{k} n^{-(2-\alpha)} \leq|z| \leq 2^{k+1} n^{-(2-\alpha)}, z \in K}\left|P_{n}\right|^{p} \leq D e^{-d p 2^{k / 2(2-\alpha)}}\left(2^{k+1} n^{-(2-\alpha)}\right)^{2} \pi .
$$

In a similar manner,

$$
\int_{|z| \leq n^{-(2-\alpha)}, z \in K}\left|P_{n}\right|^{p} \leq D\left(n^{-(2-\alpha)}\right)^{2} \pi .
$$

If we sum all these (for all $k$ ) we obtain

$$
\int_{K}\left|P_{n}\right|^{p} \leq C \frac{1}{n^{2(2-\alpha)}} .
$$

On the other hand, by the first part (part (a)) of the theorem we have

$$
\int_{K}\left|P_{n}\right|^{p} \geq c\left\|P_{n}\right\|_{K}^{p} \frac{1}{n^{2(2-\alpha)}} \geq c \frac{1}{n^{2(2-\alpha)}}
$$


with some $c>0$ (note that $P_{n}(0)=1$ ), thus, for $P_{n}$ we have the two-sided inequality

$$
\frac{1}{C} \frac{1}{n^{2(2-\alpha)}} \leq \int_{K}\left|P_{n}\right|^{p} \leq C \frac{1}{n^{2(2-\alpha)}},
$$

and this is what we wanted to get when the smallest internal angle is $<\pi$.

If the smallest internal angle at the corners of $K$ is $\geq \pi$ then $\alpha^{*}=1$, and repeat the just given proof by replacing the vertex $V$ by any point on the boundary of $K$ which is not a vertex, and to which there is a disk in the complement of $K$ which contains $V$ on its boundary. Such a $V$ and disk can be easily obtained by taking a small disk in the complement of $K$ close to a non-vertex boundary point of $K$, and moving the disk towards that point until it hits somewhere the boundary of $K$ (and the hitting point will then be $V$ ). At this $V$ the set $K$ has a "corner" of angle $\pi$. The existence of the corresponding fast decreasing polynomials (relative to $V$ and $K$ ) from (9.7) is guaranteed by Remark 5.4 (a), and the proof of (9.8) goes through with those fast decreasing polynomials without any change.

So we get in either way a sequence $\left\{P_{n}\right\}$ of polynomials such that

$$
\frac{1}{C} \frac{1}{n^{2\left(2-\alpha^{*}\right)}} \leq \int_{K}\left|P_{n}\right|^{p} \leq C \frac{1}{n^{2\left(2-\alpha^{*}\right)}}
$$

with some $C$ that depends only on $K$.

On writing this up also for $q$ instead of $p$, the inequality (9.2) follows immediately.

\section{Markov type inequalities for area measures}

In this section, we prove

Theorem 10.1 Let $K$ be as in Theorem 9.1 with piecewise $C^{1+}$ smooth boundary, and let $\alpha \pi$ be the smallest internal angle of $K$, which we assume to be positive. Then there is a constant $C$ depending only on $K$ such that for any polynomial $P_{n}$ of degree at most $n=1,2, \ldots$ we have

$$
\left\|P_{n}^{\prime}\right\|_{L^{p}(K)} \leq C n^{2-\alpha^{*}}\left\|P_{n}\right\|_{L^{p}(K)},
$$

where $\alpha^{*}=\min (\alpha, 1)$.

This estimate is sharp, for there are nonzero polynomials $P_{n}$ of degree $n=$ $1,2, \ldots$ such that

$$
\left\|P_{n}^{\prime}\right\|_{L^{p}(K)} \geq c n^{2-\alpha^{*}}\left\|P_{n}\right\|_{L^{p}(K)}
$$

with some constant $c>0$.

This is the complete analogue of the classical Markov inequality for the derivative of polynomials on the unit circle, see [7, Theorem 4.1.4]. 

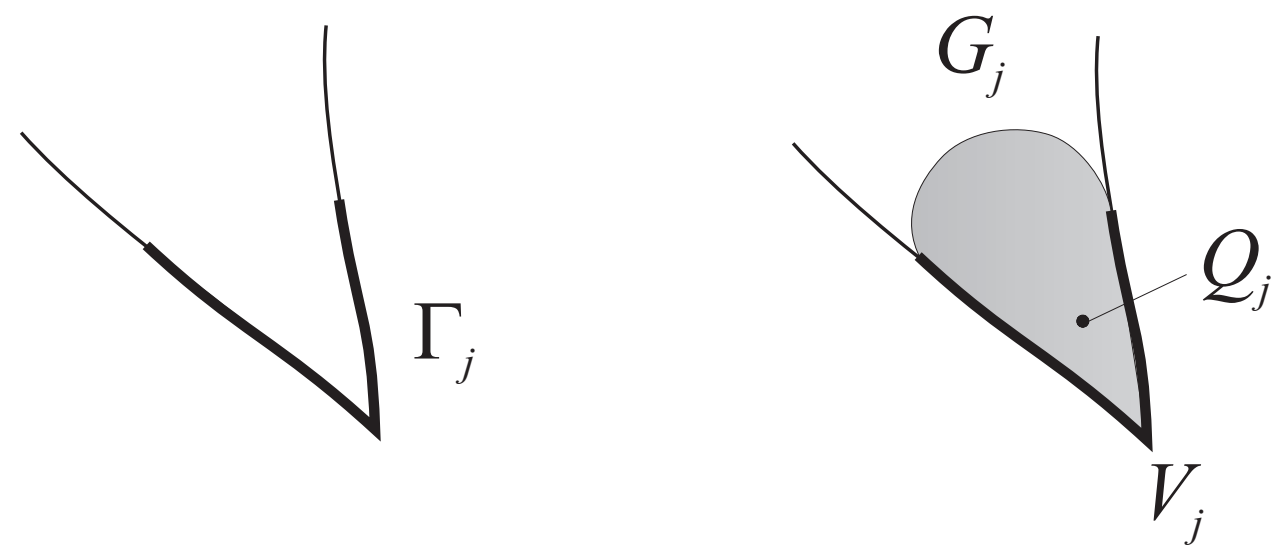

Figure 9: A typical corner, the $\operatorname{arc} \Gamma_{j}$ covering it and the domain $G_{j}$ attached to $\Gamma_{j}$

Proof of (10.1). The boundary of $K$ consist of finitely many $C^{1+}$ arcs, call the points where two such arcs are joined together a vertex. Cover the boundary $\partial K$ by finitely many small closed subarcs $\Gamma_{j}, 1 \leq j \leq m$, such that each vertex belongs to precisely one $\Gamma_{j}$. Choose domains $G_{j} \subseteq K$ such that $\bar{G}_{j} \cap \partial K=\Gamma_{j}$, and $G_{j}$ is $C^{1+}$ smooth unless one of the vertices $V$ of $K$ belongs to the (one dimensional) interior of $\Gamma_{j}$, in which case we require that $\partial G_{j}$ is a smooth arc which have both endpoints at $V$, see Figure 9 .

Select the vertex $V$ from $\partial G_{j}$ if $V$ belongs to $\Gamma_{j}$, and otherwise select a point $V$ from the (one dimensional) interior of $\Gamma_{j}$. In that latter case we can still think of $V$ as a vertex, namely with inner angle $\pi$.

Let $j$ be arbitrary, and consider the corresponding $G_{j}$ and $\Gamma_{j}$, and let $V_{j}$ be the point that is selected from $\partial G_{j}$. Then at $V_{j}$ the domain $G$ has an inner angle $\alpha_{j} \pi$ with some $0<\alpha_{j}$, but otherwise (i.e. away from $V_{j}$ ) the boundary of $G_{j}$ is $C^{1+}$ smooth. Let $Q_{j} \in G_{j}$ be a point on the the bisector of the two half tangents to $\partial G_{j}$ at the point $V_{j}$. If the subarcs $\Gamma_{j}$ are small then we can select $G_{j}$ and $Q_{j}$ in such a way that $G_{j}$ is starlike with respect to $Q_{j}$, i.e. the segment from $Q_{j}$ to any boundary point of $G_{j}$ lies inside $G_{j}$. We may also assume/achieve that the tangent lines to $G_{j}$ (half tangents at $V_{j}$ ) do not pass through the reference point $Q_{j}$.

Let $\frac{1}{2} G_{j}$ be the domain that we obtain by shrinking $G_{j}$ by a factor $1 / 2$ from the point $Q_{j}$. It is sufficient to prove that for all $j$

$$
\left\|P_{n}^{\prime}\right\|_{L^{p}\left(G_{j} \backslash \frac{1}{2} G_{j}\right)} \leq C n^{\left(2-\alpha_{j}\right)}\left\|P_{n}\right\|_{L^{p}(K)},
$$

Indeed, $n^{2-\alpha_{j}} \leq n^{2-\alpha^{*}}$ and the $G_{j} \backslash \frac{1}{2} G_{j}$ 's cover a strip $S \subset K$ attached to the boundary of $K$, hence it follows that

$$
\left\|P_{n}^{\prime}\right\|_{L^{p}(S)} \leq C n^{\left(2-\alpha^{*}\right)}\left\|P_{n}\right\|_{L^{p}(K)} .
$$


The rest of $K$, i.e. $K \backslash S$ can be covered by finitely many annuli $A_{j}$, and for each such annuli the proof of (10.4) yields

$$
\left\|P_{n}^{\prime}\right\|_{L^{p}\left(A_{j}\right)} \leq C n\left\|P_{n}\right\|_{L^{p}(K)},
$$

and (10.1) follows from these and (10.4).

For simpler notation we write $G, V, Q, \alpha$ for $G_{j}, V_{j}, Q_{j}, \alpha_{j}$, and we may also assume that $Q=0$. Let $\gamma$ be the boundary of $G, s_{\gamma}$ the arc measure on $\gamma$, and let $u \gamma$ be the $u$-homothetic copy of $\gamma$ (with respect to the origin). $\gamma$ has a parametrization $\gamma(t)=\left(\gamma_{1}(t), \gamma_{2}(t)\right), t \in[0,1], \gamma(0)=\gamma(1)=V$, where $\gamma_{j}$ is a $C^{1+}$ smooth function with $\left(\gamma_{1}^{\prime}(t)\right)^{2}+\left(\gamma_{2}^{\prime}(t)\right)^{2}>0$, and of course, $d s_{\gamma}=$ $\sqrt{\left(\gamma_{1}^{\prime}(t)\right)^{2}+\left(\gamma_{2}^{\prime}(t)\right)^{2}} d t$.

We shall use a special case of a result of V. V. Andrievskii, according to which there is a constant $C$ such that

$$
\int\left|R_{n}^{\prime}\right|^{p} d s_{\gamma} \leq C n^{(2-\alpha) p} \int\left|R_{n}\right|^{p} d s_{\gamma}
$$

for all polynomials $R_{n}$ of degree at most $n$. Indeed, this follows from [1, Theorem 1] (with $s=0$ there) if we note that in the notation of that paper in the present case $\rho_{1 / n}(z) \geq c / n^{2-\alpha}$ for all $z \in \gamma$, see Lemma 6.5 and its consequence (6.6).

Consider $\Phi(t, u)=u \gamma(t),(t, u) \in[0,1] \times\left[\frac{1}{2}, 1\right)$, which maps $[0,1] \times\left[\frac{1}{2}, 1\right)$ onto $G \backslash \frac{1}{2} G$. The absolute value of its Jacobian is

$$
|J(t, u)|=u\left|\gamma_{1}^{\prime}(t) \gamma_{2}(t)-\gamma_{2}^{\prime}(t) \gamma_{1}(t)\right|
$$

is continuous and positive. Indeed, $J(t, u)=0$ at some $t, u$ would mean that $\gamma_{1}^{\prime}(t) \gamma_{2}(t)-\gamma_{2}^{\prime}(t) \gamma_{1}(t)=0$, i.e. at $t$ the vectors $\gamma^{\prime}(t)$ and $\gamma(t)$ would be parallel. But that would mean that at $\gamma(t)$ the tangent line to $\gamma$ would pass through the origin, which is not the case by the construction of the domain $G$.

Now

$$
\begin{aligned}
\int_{G \backslash \frac{1}{2} G}\left|P_{n}^{\prime}\right|^{p} & =\int_{[0,1] \times\left[\frac{1}{2}, 1\right)}\left|P_{n}^{\prime}(u \gamma(t))\right|^{p}|J(t, u)| d t d u \\
& =\int_{1 / 2}^{1} \int_{0}^{1}\left|P_{n}^{\prime}(u \gamma(t))\right|^{p}|J(t, u)| d t d u .
\end{aligned}
$$

Here, since both $|J(t, u)|$ and $\left|\gamma^{\prime}(t)\right|$ are positive and continuous, we have

$$
\begin{aligned}
\int_{0}^{1}\left|P_{n}^{\prime}(u \gamma(t))\right|^{p}|J(t, u)| d t & \leq C \int_{0}^{1}\left|P_{n}^{\prime}(u \gamma(t))\right|^{p}\left|\gamma^{\prime}(t)\right| d t \\
& =C \int_{\gamma}\left|P_{n}^{\prime}(u z)\right|^{p} d s_{\gamma}(z)=\frac{C}{u^{p}} \int_{\gamma}\left|R_{n}^{\prime}(z)\right|^{p} d s_{\gamma}(z),
\end{aligned}
$$

where $R_{n}(z)=P_{n}(u z)$. On applying (10.5) and reversing these steps we obtain (recall that $u \in[1 / 2,1]$ )

$$
\int_{0}^{1}\left|P_{n}^{\prime}(u \gamma(t))\right|^{p}|J(t, u)| d t \leq C_{1} n^{(2-\alpha) p} \int_{0}^{1}\left|P_{n}(u \gamma(t))\right|^{p}|J(t, u)| d t .
$$


Plugging this into (10.7) we get

$$
\begin{aligned}
\int_{G \backslash \frac{1}{2} G}\left|P_{n}^{\prime}\right|^{p} & \leq C_{1} n^{(2-\alpha) p} \int_{[0,1] \times\left[\frac{1}{2}, 1\right)}\left|P_{n}(u \gamma(t))\right|^{p}|J(t, u)| d t d u \\
& =C_{1} n^{(2-\alpha) p} \int_{G \backslash \frac{1}{2} G}\left|P_{n}\right|^{p}
\end{aligned}
$$

which is (10.3).

Proof of (10.2). Let $V$ be the vertex of $K$ with smallest inner angle $\alpha \pi$, and suppose first that $\alpha<1$ (by the assumption in the theorem $\alpha>0$ ). Without loss of generality assume that $V=0$, and for this point consider the fast decreasing polynomials $P_{n}$ of Theorem 5.3 (a) with $\beta=1 / 2$ and $\gamma=\frac{1}{2(2-\alpha)}$ (see (9.7)). If $z \in K$ lies of distance $T / n^{2-\alpha}$ from 0 on the bisector of the angle at 0 , then

$$
\left|P_{n}(z)\right| \leq D \exp \left(-d\left(n\left(T / n^{2-\alpha}\right)^{\frac{1}{2-\alpha}}\right)^{1 / 2}\right) \leq \frac{1}{2}
$$

if $T$ is sufficiently large, hence on the segment $[0, z]$ there is a $w$ for which $\left|P_{n}^{\prime}(w)\right| \geq n^{2-\alpha} / 2 T$. Since $\left\|P_{n}\right\|_{K} \leq D$, the argument leading to (9.5) (or repeatedly apply (10.1)) gives that

$$
\left|P_{n}^{\prime \prime}(z)\right| \leq D e^{C_{1}} n^{2(2-\alpha)}, \quad z \in K,
$$

which in turn implies (see the argument leading to (9.6)) that

$$
\left|P_{n}^{\prime}(z)\right| \geq \frac{n^{2-\alpha}}{4 T}
$$

if $z \in K,|z-w| \leq c_{1} / n^{2-\alpha}$ and $c_{1}>0$ is sufficiently small. Since the intersection of $K$ with the set $\left\{z:|z-w| \leq c_{1} / n^{2-\alpha}\right\}$ has area measure $\geq c_{2} / n^{2(2-\alpha)}$, we obtain (cf. (9.6))

$$
\int_{K}\left|P_{n}^{\prime}\right|^{p} \geq c_{2} n^{(2-\alpha)(p-2)} .
$$

Since by (9.9)

$$
\int_{K}\left|P_{n}\right|^{p} \leq C n^{-2(2-\alpha)},
$$

the inequality (10.2) follows.

If the smallest internal angle at the corners of $K$ is $\geq \pi$ then $\alpha^{*}=1$, and repeat the just given proof by replacing the vertex $V$ by any point on the boundary of $K$ which is not a vertex, but for which there is a disk in the complement of $K$ that contains $V$ on its boundary (see the end of the proof of (9.2) in the preceding section). 


\section{Christoffel functions}

For a Borel-measure $\mu$ on the plane with compact support the $n$-th Christoffel function is defined as

$$
\lambda_{n}(\mu, z)=\inf _{P_{n}(z)=1} \int\left|P_{n}\right|^{2} d \mu,
$$

where the infimum is taken for all polynomials $P_{n}$ of degree at most $n$ that take the value 1 at the point $z$. These play a significant role in the theory of orthogonal polynomials, see the papers [16] and [22] for their applications. If $p_{n}$ denote the orthonormal polynomials with respect to $\mu$, then we have the formula

$$
\frac{1}{\lambda_{n}(\mu, z)}=\sum_{k=0}^{n}\left|p_{k}(z)\right|^{2}
$$

Orthogonal polynomials with respect to area measures go back to Carleman [5]. If $\left.A\right|_{K}$ denotes the area measure on some closed Jordan domain $K$, then inside the domain the Christoffel functions with respect to $\left.A\right|_{K}$ lie in between two positive constants independent of $n$ (but depending on the position of the point $z$ inside the domain), while outside the domain they are exponentially small in $n$. This sharp decrease in the Christoffel functions around the boundary has recently been used for a domain reconstruction procedure in the paper [9]. On the boundary of the domain the behavior of the $n$-th Christoffel function is typically like a negative power of $n$, and the results from previous section easily allow us to determine that power. For illustration we shall do that at a corner.

Theorem 11.1 Let the closed Jordan domain $K$ have a $C^{1+}$ smooth corner of inner angle $\alpha \pi$ with $0<\alpha \leq 1$ at the point $z_{0}$. Then

$$
\frac{c}{n^{2(2-\alpha)}} \leq \lambda_{n}\left(\left.A\right|_{K}, z_{0}\right) \leq \frac{C}{n^{2(2-\alpha)}}, \quad n=1,2, \ldots
$$

with some constants $0<c, C$.

The same result holds if $K$ is the union of finitely many closed Jordan domains.

Proof. Assume $z_{0}=0$.

Let $K_{1} \subset K$ be a compact subset such that $K_{1}$ coincides with $K$ in a neighborhood of 0 , but otherwise, i.e. away from 0 , it has $C^{1+}$ smooth boundary. If we apply Theorem 9.1 with $p=2$ and $q=\infty$ to the domain $K_{1}$, then it follows that if $P_{n}(0)=1$, then

$$
\int_{K}\left|P_{n}\right|^{2} d A \geq \int_{K_{1}}\left|P_{n}\right|^{2} \geq \frac{c}{n^{2(2-\alpha)}}
$$

with some constant $c$ depending only on $K_{1}$, which proves the lower estimate in (11.2). 
To prove the upper inequality, let now $K \subset K_{2}$ be a compact set such that $K_{2}$ coincides with $K$ in a neighborhood of 0 , but otherwise it has $C^{1+}$ smooth boundary. If we consider for $K_{2}$ the fast decreasing polynomials $P_{n}$ used in the proof of Theorem 9.1 with the properties (9.7), then we have (see (9.8) for $K_{2}$ replacing $K$ there)

$$
\int_{K}\left|P_{n}\right|^{2} d A \leq \int_{K_{2}}\left|P_{n}\right|^{2} d A \leq \frac{C}{n^{2(2-\alpha)}},
$$

and this proves the upper estimate in (11.2).

In view of (11.1) one easily gets the following estimates for the orthonormal polynomials $p_{n}(z)$ with respect to area measures on $K$ : under the assumptions of Theorem 11.1 we have with some constant $C$

$$
\left|p_{n}\left(z_{0}\right)\right| \leq C n^{2-\alpha},
$$

and for infinitely many $n$

$$
\left|p_{n}\left(z_{0}\right)\right| \geq C n^{3 / 2-\alpha} .
$$

Away from the corners more precise results can be found in the paper [24] for domains with piecewise analytic boundary.

Acknowledgement. The first author was supported by NSF DMS-1265375. The second author's work was partially supported by the European Union and the State of Hungary, co-financed by the European Social Fund in the framework of TÁMOP 4.2.4. A/2-11-1-2012-0001 National Excellence Program.

\section{References}

[1] V. V. Andrievskii, Weighted $L_{p}$ Bernstein-type inequalities on a quasismooth curve in the complex plane, Acta Math. Hungar. 135(2011), 8-23.

[2] V. V. Andrievskii, V. I. Belyi and V. K. Dzjadyk, Conformal Invariants in Constructive Theory of Functions of Complex Variable, World Federation Publisher, Atlanta, 1995.

[3] V. V. Andrievskii, Chebyshev polynomials on a system of continua, Constructive Approximation (to appear)

[4] D. H. Armitage and S. J. Gardiner, Classical Potential Theory, Springer Verlag, Berlin, Heidelberg, New York, 2002.

[5] T. Carleman, Über die Approximation analytischer Funktionen durch lineare Aggregate von vorgegebenen Potenzen, Ark. Mat. Astr. Fys., 17(1923), 215-244. 
[6] J. B. Conway Functions of one complex variable, Springer-Verlag (New York-Heidelberg-Berlin 1973).

[7] R. A. DeVore and G. G. Lorentz, Constructive Approximation, Grundlehren der mathematischen Wissenschaften, 303, Springer-Verlag, Berlin, Heidelberg, New York 1993.

[8] J. B. Garnett and D. E. Marshall, Harmonic measure, Cambridge University Press, New mathematical monographs, Cambridge, New York, 2005.

[9] B. Gustafsson, M. Putinar, E. Saff, and N. Stylianopoulos, Bergman polynomials on an archipelago: Estimates, zeros and shape reconstruction, $A d$ vances in Math., 222(2009), 1405-1460.

[10] K.G. Ivanov and V. Totik, Fast decreasing polynomials, Constructive Approximation, 6(1990), 1-20.

[11] K. Ivanov, P. Petrushev and Y. Xu, Decomposition of spaces of distributions induced by tensor product bases, J. Funct. Anal., 263(2012), 11471197.

[12] K. Ivanov, P. Petrushev and Y. Xu, Sub-exponentially localized kernels and frames induced by orthogonal expansions, Math. Z., 264(2010), 361-397.

[13] R. S. Lehman, Development of the mapping function at an analytic corner, Pacific J. Math., 7(1957), 1437-1449.

[14] E. Levin and D. S. Lubinsky, Orthogonal polynomials for exponential weights, CMS Books in Mathematics/Ouvrages de Mathmatiques de la SMC, 4. Springer-Verlag, New York, 2001.

[15] B. Nagy and V. Totik, Sharpening Hilbert's lemniscate theorem, J. Anal. Math. 96 (2005), 191-223.

[16] P. Nevai, Géza Freud, orthogonal polynomials and Christoffel functions. A case study, J. Approx. Theory, 48(1986), 1-167.

[17] R. Nevanlinna, Analytic Functions, Grundlehren der mathematischen Wissenschaften, 162, Springer Verlag, Berlin, 1970.

[18] C. Pommerenke, Univalent Functions, Vandenhoeck und Ruprecht, Gottingen, 1975.

[19] Ch. Pommerenke, Boundary Behavior of Conformal Mappings, Grundlehren der mathematischen Wissenschaften, 299, Springer Verlag, Berlin, Heidelberg New York, 1992.

[20] T. Ransford, Potential Theory in the Complex Plane, Cambridge University Press, 1995. 
[21] E. B. Saff and V. Totik, Logarithmic Potentials with External Fields, Grundlehren der mathematischen Wissenschaften, 316, Springer Verlag, Berlin, Heidelberg, 1997.

[22] B. Simon, The Christoffel-Darboux kernel, "Perspectives in PDE, Harmonic Analysis and Applications" in honor of V.G. Maz'ya's 70th birthday, to be published in Proceedings of Symposia in Pure Mathematics, 79(2008), 295-335.

[23] M. L. Sodin and P. M. Yuditskii, Functions least deviating from zero on closed subsets of the real axis, Algebra $i$ Analiz, 4(1992), 1-61; English transl. in St. Petersburg Math. J., 4(1993), 201-249.

[24] N. Stylianopoulos, Strong Asymptotics for Bergman Polynomials over Domains with Corners and Applications, Constructive Approx., 38(2013), 59100.

[25] V. Totik, Christoffel functions on curves and domains, Trans. Amer. Math. Soc., 362 (2010), 2053-2087.

[26] Totik V., Szegö's problem on curves Amer. J. Math., 135 (2013), 15071524.

[27] V. Totik, Chebyshev polynomials on a systems of curves, J. d'Analyse Math., 118(2012), 317-338.

[28] V. Totik, Chebyshev polynomials on compact sets, Potential Analysis, 40(2014), 511-524.

[29] T. Varga, Christoffel functions for doubling measures on quasismooth curves and arcs, Acta Math. Acad. Sci. Hungar., 141 (2013), 161-184.

[30] J. L. Walsh, Interpolation and Approximation by Rational Functions in the Complex Domain, fifth edition, Amer. Math. Soc. Colloquium Publications, XX, Amer. Math. Soc., Providence, 1969.

[31] H. Widom, Extremal polynomials associated with a system of curves in the complex plane, Adv. Math., 3(1969), 127-232.

Vilmos Totik

Bolyai Institute

MTA-SZTE Analysis and Stochastics Research Group

University of Szeged

Szeged

Aradi v. tere 1, 6720, Hungary

and

Department of Mathematics and Statistics

University of South Florida

4202 E. Fowler Ave, CMC342 
Tampa, FL 33620-5700, USA

totik@mail.usf.edu

Tamás Varga

Potential Analysis Research Group, ERC Advanced Grant No. 267055

Bolyai Institute

University of Szeged

Szeged

Aradi v. tere 1, 6720, Hungary

vargata@math.u-szeged.hu 